\title{
High precision Monte Carlo radiative transfer in dusty media
}

\author{
G. Niccolini ${ }^{1}$, P. Woitke ${ }^{2}$, and B. Lopez ${ }^{1}$ \\ 1 Observatoire de la Côte d'Azur, Département Fresnel, UMR 6528, BP 4229, 06034 Nice Cedex 4, France \\ 2 Zentrum für Astronomie und Astrophysik, TU Berlin, Hardenbergstraße 36, 10623 Berlin, Germany
}

Received 19 February 2002 / Accepted 19 November 2002

\begin{abstract}
In this paper, we present a refined Monte Carlo method for the solution of multi-dimensional radiative transfer problems in dusty media, for instance in the circumstellar envelopes of AGB and post AGB stars. In order to push the performance of the Monte Carlo method, we have included several basic improvements of the standard procedure which avoid the usual increase of the noise level in extremely optically thin or optically thick situations. The improvements comprise an accurate temperature determination scheme, e.g. based on the calculation of mean intensities, and an inclusion of several deterministic elements for the treatment of the absorption during the photon propagation. The method is capable of accurately modelling discontinuous opacity structures, such as clumpy media, and is applicable in a broad range of optical depths. It can provide the gas and dust temperature structures required for a more complex physical modelling, e.g. hydrodynamics, chemistry or dust formation. It may be used to predict colours, spectral energy distributions, visibilities and monochromatic images. The paper is completed by an exploration of the influence of dust clouds and small holes in dust shells on the spectral energy distribution and their optical appearance in monochromatic images. The underlying temperature structures are discussed.
\end{abstract}

Key words. radiative transfer - methods: numerical - stars: circumstellar matter

\section{Introduction}

Numerous high-angular resolution observations obtained in the past decade (e.g. O'Dell \& Handron 1996; Shara et al. 1997; Weigelt et al. 1998; Haniff \& Buscher 1998; Tuthill et al. 2000) have accumulated convincing evidence that astrophysical gases often reveal an internal, inhomogeneous structure on various length scales. The next generation of observational instruments, like the Vlti, Sofia, Lbt, Ngst, and Alma, can be expected to reveal even more details. In order to interpret these new observational results, likewise detailed, multi-dimensional radiative transfer methods must be developed which can handle e.g. clumpy media and/or complex spatial opacity structures.

The performance of multi-dimensional radiative transfer codes has thus become one of the limiting factors in contemporary theoretical astrophysics. In many astrophysical situations, the radiation field is strongly coupled to other physical processes. When considering for instance the strong temperaturedependence of the process of dust formation around AGB stars (Sedlmayr 1994; Woitke 2001) or hydrodynamical models of stellar atmospheres (e.g. Asplund et al. 2000), the need of accurate multi-dimensional radiative transfer becomes obvious.

The Monte Carlo method provides an interesting, alternative approach for the solution of various (continuum and line) radiative transfer problems as compared to deterministic methods. Monte Carlo methods are simple in concept, easy to

Send offprint requests to: G. Niccolini, e-mail: nicolin@obs-nice.fr understand, and flexible. Advantages of the Monte Carlo methods especially arise in cases of a complex distribution of matter in space (e.g. clumpy medium, Spaans 1996; Och et al. 1998) or in case of difficult inner or outer boundary conditions (e.g. interstellar medium irradiated by stellar clusters, Gordon et al. 2001), whenever the spatial and angular resolution in the models is of crucial importance.

The degree of sophistication in the Monte Carlo methods (concerning spatial symmetry, inclusion of multi-scattering processes, polarisation effects, etc.) has continuously improved, according to the increasing computer facilities. Fields of astrophysical application reach from classical stellar atmospheres (Lucy et al. 1999) over circumstellar dust shells (Lefèvre et al. 1982; Yusef-Zadeh \& Morris 1984), young stellar objects and active galactic nuclei (Fischer et al. 1994; Wolf et al. 1999), star formation (Hogerheidje \& van der Tak 2000) to interstellar medium and galaxies (Gordon et al. 2001). One of the principal disadvantages of the Monte Carlo methods, however, is the presence of a random scatter in the results (the Monte Carlo noise). Although this noise naturally vanishes with increasing number of photon packages used, the reduction of the noise to a prescribed level can make a Monte Carlo method very time-consuming, thereby limiting its range of practical applicability. Deterministic elements in the Monte Carlo experiment can be included in order to reduce this noise, for instance by forcing an interaction, e.g. the "forced first collision" introduced by Cashwell \& Everett (1959) or the use of "weighted photons" (Witt 1977). 
Another basic problem of the Monte Carlo methods is the limited range of optical depth to which it can be applied. The standard approach to Monte Carlo method considers packages of photon energy that perform a random walk through a model volume guided by absorption and scattering events. If the medium is very optically thin $(\tau \rightarrow 0)$, interactions are rare and only little information is produced by the Monte Carlo experiment. The Monte Carlo noise is consequently increased for quantities directly related to this interaction (e.g. local absorption and radiation field, etc.).

In contrast, if the medium becomes very optically thick $(\tau \rightarrow \infty)$, photons emitted in a small part of the model volume are scattered and interact so often that they practically never leave this part. This also reduces the gain of useful information produced by the Monte Carlo experiment, that is the exchange of energies between different parts of the model volume.

In this paper ${ }^{1}$, we present a refined Monte Carlo method in media with temperature-independent opacities (e.g. dusty media) which combines some improvements of the standard procedure in order to avoid the usual increase of the noise level in optically thin and optically thick situations. The work is based on the Monte Carlo code originally developed by Lopez et al. (1995). The increase of the performance for optically thin problems is achieved by calculating the temperature structure on the basis of mean intensity as proposed by Lucy (1999) (see Sect. 3.3.2). Optically thick situations are tackled by the inclusion of several deterministic elements for the treatment of the absorption, forced all along the rays (see Sect. 3.3.3). The temperature structure of the medium in radiative equilibrium is found afterwards by applying iterative techniques (Sect. 4). This procedure seems to be advantageous for our purpose as compared to other techniques which fulfil the constraint of radiative equilibrium automatically (e.g. Lucy 1999; Bjorkman $\&$ Wood 2001).

In Sect. 5, we present some spherically symmetric test calculations which demonstrate the achieved accuracy in comparison to analytical solutions or solutions obtained with a deterministic code. Sections 6.1 and 6.2 consider simple prototypes of a clumpy circumstellar environment, namely an isolated optically thick dust cloud and a hole in an optically thick dust shell. We discuss the obtained temperature structures, the calculated spectral energy distributions and spectral images. Section 7 comprises our conclusions and future aims.

\section{The physical problem}

\subsection{The radiative transfer problem}

We consider an irradiated volume $V$ of matter in which we aim at a solution of the time-independent radiative transfer equation

$\frac{\mathrm{d}}{\mathrm{d} s} I_{\lambda}(\boldsymbol{r}, \widehat{\boldsymbol{n}})=-\kappa_{\lambda}^{\mathrm{ext}}(\boldsymbol{r}) I_{\lambda}(\boldsymbol{r}, \widehat{\boldsymbol{n}})+\eta_{\lambda}(\boldsymbol{r}, \widehat{\boldsymbol{n}})$

where $I_{\lambda}(\boldsymbol{r}, \widehat{\boldsymbol{n}})$ is the specific intensity at position $\boldsymbol{r}$ in direction of the unit vector $\widehat{\boldsymbol{n}}$ at wavelength $\lambda$ and $s$ denotes the distance

\footnotetext{
1 The interested reader can find a more detailed version of the paper at http://astro.physik.tu-berlin.de/ woitke/ MonteCarlo.ps.gz
}

along the ray from a starting point $\boldsymbol{r}_{0}$ located at the surface of $V$, i.e. $\boldsymbol{r}=\boldsymbol{r}_{0}+s \widehat{\boldsymbol{n}}$.

Assuming local thermodynamic equilibrium (LTE) and coherent scattering, the total emissivity $\eta_{\lambda}(\boldsymbol{r}, \widehat{\boldsymbol{n}})$ is given by

$$
\begin{aligned}
\eta_{\lambda}(\boldsymbol{r}, \widehat{\boldsymbol{n}})= & \kappa_{\lambda}^{\mathrm{abs}}(\boldsymbol{r}) B_{\lambda}(T(\boldsymbol{r})) \\
& +\kappa_{\lambda}^{\mathrm{sca}}(\boldsymbol{r}) \frac{1}{4 \pi} \iint g_{\lambda}\left(\boldsymbol{r}, \widehat{\boldsymbol{n}}^{\prime}, \widehat{\boldsymbol{n}}\right) I_{\lambda}\left(\boldsymbol{r}, \widehat{\boldsymbol{n}}^{\prime}\right) \mathrm{d}^{2} \widehat{\boldsymbol{n}}^{\prime},
\end{aligned}
$$

where $B_{\lambda}$ is the Planck function and $T(\boldsymbol{r})$ the temperature of the medium at $\boldsymbol{r}$, which is assumed to be unique ${ }^{2}$, and $g_{\lambda}\left(\boldsymbol{r}, \widehat{\boldsymbol{n}}^{\prime}, \widehat{\boldsymbol{n}}\right)$ is the phase function. The quantities $\kappa_{\lambda}^{\mathrm{abs}}, \kappa_{\lambda}^{\mathrm{sca}}$ and $\kappa_{\lambda}^{\mathrm{ext}}=\kappa_{\lambda}^{\mathrm{abs}}+\kappa_{\lambda}^{\text {sca }}$ are the coefficients for true absorption, scattering and extinction, respectively.

The matter in the volume $V$ is assumed to be in radiative equilibrium which provides an integral auxiliary condition for the solution of Eq. (1)

$\int_{0}^{\infty} \kappa_{\lambda}^{\mathrm{abs}}(\boldsymbol{r}) B_{\lambda}(T(\boldsymbol{r})) \mathrm{d} \lambda=\int_{0}^{\infty} \kappa_{\lambda}^{\mathrm{abs}}(\boldsymbol{r}) J_{\lambda}(\boldsymbol{r}) \mathrm{d} \lambda$,

where $J_{\lambda}(\boldsymbol{r})$ is the mean intensity.

The aims of the calculations are to obtain

- the internal temperature structure of the matter $T(\boldsymbol{r})$ and the internal radiation field, e.g. $J_{\lambda}(\boldsymbol{r})$, for $\boldsymbol{r} \in V$. These quantities can be used as necessary input for other physical modelling, e.g. time-dependent chemistry, hydrodynamics and/or dust formation;

- the specific intensities $I_{\lambda}\left(\boldsymbol{r}_{0}+s_{\max } \widehat{\boldsymbol{n}}, \widehat{\boldsymbol{n}}\right)$ escaping from the system, where $s_{\max }$ denotes the distance where the rays exits $V$. From these intensities, the observable flux in different view directions (spectra, SED) and monochromatic images at different wavelengths can be calculated.

\subsection{Dust opacities}

In this paper we will focus on media, in which the opacity of small solid particles (dust grains) strongly dominates and gas opacities can be neglected. The dust grains are assumed to be spherical. The opacities are related to an arbitrary size distribution function of dust grains $f(a, r)\left[\mathrm{cm}^{-4}\right]$ via

$$
\begin{aligned}
& \kappa_{\lambda}^{\mathrm{abs}}(\boldsymbol{r})=\int_{a_{\min }}^{a_{\max }} \pi a^{2} Q_{\mathrm{abs}}(a, \lambda) f(a, \boldsymbol{r}) \mathrm{d} a \\
& \kappa_{\lambda}^{\text {sca }}(\boldsymbol{r})=\int_{a_{\min }}^{a_{\max }} \pi a^{2} Q_{\mathrm{sca}}(a, \lambda) f(a, \boldsymbol{r}) \mathrm{d} a
\end{aligned}
$$

where $a_{\min }$ and $a_{\max }$ are the minimum and maximum grain size respectively.

For spherical particles, the general angular dependence of the phase function $g_{\lambda}(\boldsymbol{r}, \widehat{\boldsymbol{n}}, \widehat{\boldsymbol{n}})$ simplifies to a dependence on the

\footnotetext{
2 In Eq. (2) we treat the matter at $r$ as a one component medium with a unique temperature. Effects caused by different grain temperatures at $\boldsymbol{r}$ are ignored.
} 
scattering angle $\cos \vartheta=\widehat{\boldsymbol{n}} \cdot \widehat{\boldsymbol{n}}$

$g_{\lambda}(\boldsymbol{r}, \vartheta)=\frac{4 \pi}{\kappa_{\lambda}^{\mathrm{sca}}(\boldsymbol{r})} \int_{a_{\min }}^{a_{\max }} S(a, \lambda, \vartheta) f(a, \boldsymbol{r}) \mathrm{d} a$.

The dimensionless dust absorption and scattering efficiencies, $Q_{\mathrm{abs}}(a, \lambda)$ and $Q_{\mathrm{sca}}(a, \lambda)$, as well as the amplitude function $S(a, \lambda, \vartheta)$ can be computed by Mie theory, e.g. applying the algorithm of Wiscombe (1980), on the basis of the refractory index of the dust grain material.

\section{The Monte Carlo method}

The basic idea of the Monte Carlo method is to split the emergent radiative energies into "Photon Packages" (PP), which perform a random walk through the model volume. In this paper, we consider the astrophysical situation of a star within a dust envelope which is subdivided into a grid of spatial cells. The PP occasionally interact with the matter in the cells, where they are (partly) absorbed and possibly change their propagation direction due to scattering events. The propagation of the $\mathrm{PP}$ produces the necessary informations needed to solve the radiative transfer problem in a probabilistic way.

Consequently, the results of the Monte Carlo method are naturally subject to a random scatter, which can limit the range of applicability of the method quite substantially. Hereafter, we present several improvements about how to reduce this noise and so to push the performance of a Monte Carlo method. The methods presented in this paper can be summarised as follows:

- independent creation of stellar and cell PPs;

- deterministic choice of wavelength and monochromatic luminosity of the PPs;

- random choice of initial point and the initial emission direction of the PPs as well as random choice of the next interaction point;

- inclusion of deterministic elements during the photon propagation phase;

- determination of the radiative equilibrium temperature structure after the propagation phase has been completed.

\subsection{Spatial grid and symmetry}

For the remainder of this paper, we consider the twodimensional case of axisymmetry. A cell is defined as

$r \in\left[r_{n}, r_{n+1}\right] n=1, \ldots, N_{r}$

$\theta \in\left[\theta_{k}, \theta_{k+1}\right] k=1, \ldots, N_{\theta}$

$\phi \in[0,2 \pi)$,

where $r, \theta$ and $\phi$ are spherical coordinates. The extension to 3D is straightforward, introducing cell boundaries also or the longitude angle $\phi$. In axisymmetry, we may refer to a particular cell by specifying its radial and angular indices, $n$ and $k$ respectively, abbreviated by a multi-index $\xi=(n, k)$. Further assumptions about the physical quantities within the cells are as follows:

- the temperature $T(\boldsymbol{r})$ is constant in each cell;
- the extinction, absorption and scattering mass coefficients $\hat{\kappa}_{\lambda}^{\text {ext }}, \hat{\kappa}_{\lambda}^{\text {abs }}$ and $\hat{\kappa}_{\lambda}^{\text {sca }}$, respectively, are constant throughout one cell, i.e. $\kappa_{\lambda}^{\mathrm{ext}}(\boldsymbol{r})=\rho(\boldsymbol{r}) \hat{\kappa}_{\lambda}^{\mathrm{ext}}(\xi)$. However, the mass density $\rho(\boldsymbol{r})$ may have an arbitrary spatial dependence;

- the albedo $\gamma_{\lambda}(\boldsymbol{r})=\kappa_{\lambda}^{\mathrm{sca}}(\boldsymbol{r}) / \kappa_{\lambda}^{\mathrm{ext}}(\boldsymbol{r})$ and the phase function $g_{\lambda}(\boldsymbol{r}, \vartheta)$ are constant in each cell.

\subsection{Photon package generation}

The wavelength and the initial luminosity of a particular PP are chosen in a deterministic way. For each considered wavelength point $\lambda_{i}\left(i=1 \ldots N_{\lambda}\right)$, the monochromatic luminosity of the star or the considered cell ${ }^{3}$ is subdivided into $N_{i}^{x}$ packages of equal energy content

$p_{\lambda_{i}}=\frac{L_{x}\left(\lambda_{i}\right)}{N_{i}^{x}}$

with general emitter index $x=\{\star, \xi\}$, i.e. $\star$ and $\xi$ mark the star and the cell of index $\xi$, respectively.

The star is assumed to radiate as a blackbody of temperature $T_{\star}$. Consequently, the stellar luminosity is

$L_{\star}\left(\lambda_{i}\right)=4 \pi^{2} R_{\star}^{2} B_{\lambda_{i}}\left(T_{\star}\right)$.

The luminosity of cell $\xi$ is given in LTE by

$L_{\xi}\left(\lambda_{i}\right)=4 \pi \hat{\kappa}_{\lambda_{i}}^{\mathrm{abs}}(\xi) B_{\lambda_{i}}\left(T_{\xi}\right) \int_{\mathrm{V}(\xi)} \rho(\boldsymbol{r}) \mathrm{d} V$

where $V(\xi)$ is the volume of cell $\xi$.

The number of photon packages $N_{i}^{x}$ may depend on the wavelength and may be freely chosen according to the purpose and the desired accuracy of the model. For instance, the calculation of the Spectral Energy Distribution (SED) requires a constant $N_{i}^{x}$ to keep the Monte Carlo noise on a similar level for all wavelengths. However, it is more advantageous to keep $w_{i} p_{\lambda_{i}}$ constant for the temperature determination, $w_{i}$ being the wavelength integration weight (see Sect. 4).

The initial position $\boldsymbol{r}_{0}$ of a stellar PP is chosen uniformly distributed onto the stellar surface by means of two equally distributed (pseudo) random numbers $z_{1}, z_{2} \in[0,1)$ (Cashwell \& Everett 1959, see Knuth 1981 for an alternative way)

$\cos \vartheta_{\star}=1-2 z_{1}$

$\varphi_{\star}=2 \pi z_{2}$.

In the case of cell PPs, we must assure that the opacity distribution in the cell $\xi$, and therewith the emissivity of the matter in LTE, is properly resembled by the Monte Carlo experiment. Since we allow the mass density to vary inside one cell, the choice of a cell starting point is not straightforward and requires special efforts (see Appendix A).

Assuming that the emitted stellar intensities $I_{\lambda}^{\star}$ are independent of $\theta_{\mathrm{e}}^{\star}$ and $\phi_{\mathrm{e}}^{\star}$ (see Fig. 1), as is true for a blackbody sphere ${ }^{4}$, the initial direction of a stellar photon package

\footnotetext{
3 The radiant emission from the matter inside the model volume is considered cell by cell.

${ }^{4}$ Other stellar irradiation laws (using e.g. the results of stellar atmosphere calculations) or limb darkening laws may be considered straightforwardly, provided that $I_{\lambda}^{\star}\left(\theta_{\mathrm{e}}^{\star}\right)$ is known for each wavelength.
} 


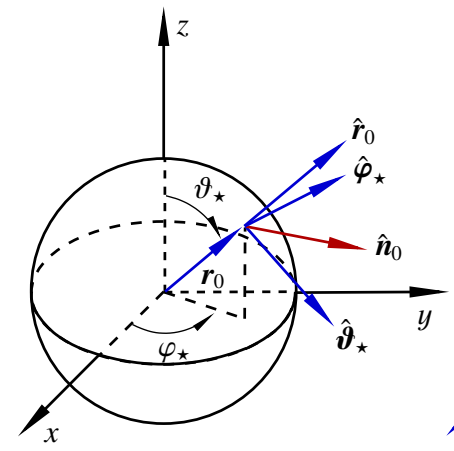

(a)

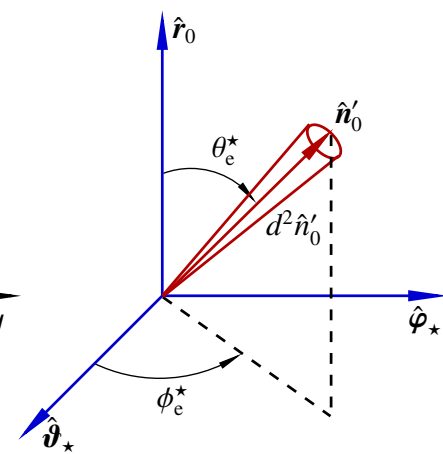

(b)
Fig. 1. a) Choice of the the emission point $\boldsymbol{r}_{0}$ on the stellar surface. b) Choice of the emission direction $\widehat{\boldsymbol{n}}_{0}$.

$\widehat{\boldsymbol{n}}_{0}^{\prime}$ is generated by means of two further random numbers $z_{3}, z_{4} \in[0,1)$ (Cashwell \& Everett 1959)

$\sin \theta_{\mathrm{e}}^{\star}=\sqrt{z_{3}}$

$\phi_{\mathrm{e}}^{\star}=2 \pi z_{4}$.

Finally, the initial direction of the stellar photon package in Cartesian coordinates, $\widehat{\boldsymbol{n}}_{0}$, is given by the matrix product with an appropriate rotation matrix.

Assuming the cell emission to be isotropic, the choice of the initial emission direction $\widehat{\boldsymbol{n}}_{0}$ of a cell PP is made analogous to the stellar emission point (Eqs. (13) and (14)).

\subsection{Photon propagation}

We describe here two distinct propagation methods. Method 1 is based on the determination of mean intensities along the photon trajectories as proposed by Lucy (1999), which is more suitable for optically thin cases, because the mean intensities, and consequently the temperatures, can be computed even in the limiting case of empty cells.

In method 2, we treat the absorption in a deterministic way according to the concept of Cashwell \& Everett (1959), which will be preferred in optically thick situations, because the PPs are forced to be partly absorbed all along the ray, i.e. they produce in any case at least some useful information, even if the medium is very optically thick.

\subsubsection{Optical depth scale}

In both methods, the integration of the optical depth $\tau_{\lambda}(s)$ along direction $\widehat{\boldsymbol{n}}$ from a starting point $\boldsymbol{r}_{0}$ with increasing distance $s$ is carried out cell by cell as encountered along the ray. It is essential to exactly trace possible $\kappa_{\lambda}^{\text {ext }}$-discontinuities at the cell boundaries. Otherwise, the numerical inversion of $\tau_{\lambda}(s)$ close to a cell boundary might result in the choice of an interaction point in a "wrong" cell". Therefore, the distance to the next cell boundary must be determined with high precision,

\footnotetext{
5 This could lead to spurious effects. For example, a wrong absorption event in an practically "empty" cell in radiative equilibrium (with a very low $\kappa_{\lambda}^{\mathrm{abs}}$ ) must be compensated by the same amount by thermal emission, leading by error to very high temperatures.
}

which is actually one of the most time-consuming parts of our Monte Carlo code.

Starting from $\boldsymbol{r}_{0}$, the spatial position along the ray changes as $\boldsymbol{r}=\boldsymbol{r}_{0}+s \widehat{\boldsymbol{n}}$. The extinction coefficient $\kappa_{\lambda}^{\mathrm{ext}}(s)=\kappa_{\lambda}^{\mathrm{ext}}\left(\boldsymbol{r}_{0}+s \widehat{\boldsymbol{n}}\right)$ is evaluated at several sampling points $\boldsymbol{r}_{j}=\boldsymbol{r}_{0}+s_{j} \widehat{\boldsymbol{n}},(0 \leq j \leq$ $n)$. For each elementary integration step, $j \rightarrow j+1$, $\kappa_{\lambda}^{\mathrm{ext}}(s)$ is approximated with a second order discretisation scheme

$\kappa_{\lambda}^{\mathrm{ext}}(s) \approx a_{j} s^{2}+b_{j} s+c_{j}$.

The optical depth at point $\boldsymbol{r}_{j}$ is then computed with the quadrature formula

$\tau_{\lambda}^{j}=\int_{0}^{s_{j}} \kappa_{\lambda}^{\mathrm{ext}}\left(\boldsymbol{r}_{0}+s \widehat{\boldsymbol{n}}\right) \mathrm{d} s \approx \sum_{j=1}^{n} w_{j} \kappa_{\lambda}^{\mathrm{ext}}\left(s_{j}\right)$,

where the integration weights $w_{j}$ are computed by utilising Eq. (17). The crossing of a cell boundary, say at step $J$, is finally achieved by making a tiny step into the next cell $s_{J+1}=$ $s_{J}+\epsilon$ for which the increment of the optical depth is neglected $\tau_{\lambda}{ }^{J+1}=\tau_{\lambda}{ }^{J}$.

The result of the integration along a particular ray is a vector of positions $\boldsymbol{r}_{0}, \boldsymbol{r}_{1}, \ldots, \boldsymbol{r}_{n}$ and associated optical depths $\tau_{\lambda}{ }^{0}=0, \tau_{\lambda}^{1}, \ldots, \tau_{\lambda}{ }^{n}$.

\subsubsection{Method 1}

In this method, the absorption, extinction and scattering of the photon package is treated in a probabilistic way. The optical depth of the next interaction point $\tau_{\lambda}$ is chosen randomly according to

$\tau_{\lambda}=-\ln (1-z)$,

where $z \in[0,1)$ is an equally distributed random number (see Cashwell \& Everett 1959).

Up to the next interaction point $\boldsymbol{r}$, useful information about the passage of photon energy through the cells is automatically created by the Monte Carlo experiment (see Fig. 2). Following Lucy (1999), this information can be used to calculate the mean intensity $J_{\lambda}$ in the cells, which provides the basis for temperature determination in this method. The PPs contribute to the mean intensity according to their energy times their pathlength through the cells

$j_{\lambda}\left(x^{\prime}, \xi\right) \leftarrow j_{\lambda}\left(x^{\prime}, \xi\right)+p_{\lambda} \Delta s$,

where $j_{\lambda}\left(x^{\prime}, \xi\right)$ is the contribution of the PPs emitted from $x^{\prime}$, i.e. from the star $\left(x^{\prime}=\star\right)$ or from the cell with index $\left(x^{\prime}=\xi^{\prime}\right)$, to the mean intensity $J_{\lambda}$ in cell $\xi$. The information is incremented whenever a photon package crosses a cell boundary and thus exits a cell $\xi$, or when the package interacts with the matter in cell $\xi . \Delta s$ is the travelled distance through cell $\xi$ or the distance up to the interaction point in cell $\xi$, respectively.

If the optical depths $\tau_{\lambda}$ exceeds the maximum value at the end of the ray $\tau_{\lambda}^{n}$, the photon package leaves the model volume and consequently contributes to the observable flux in direction $\widehat{\boldsymbol{n}}$

$f_{\lambda}\left(x^{\prime}, j_{\theta}\right) \leftarrow f_{\lambda}\left(x^{\prime}, j_{\theta}\right)+p_{\lambda}$. 


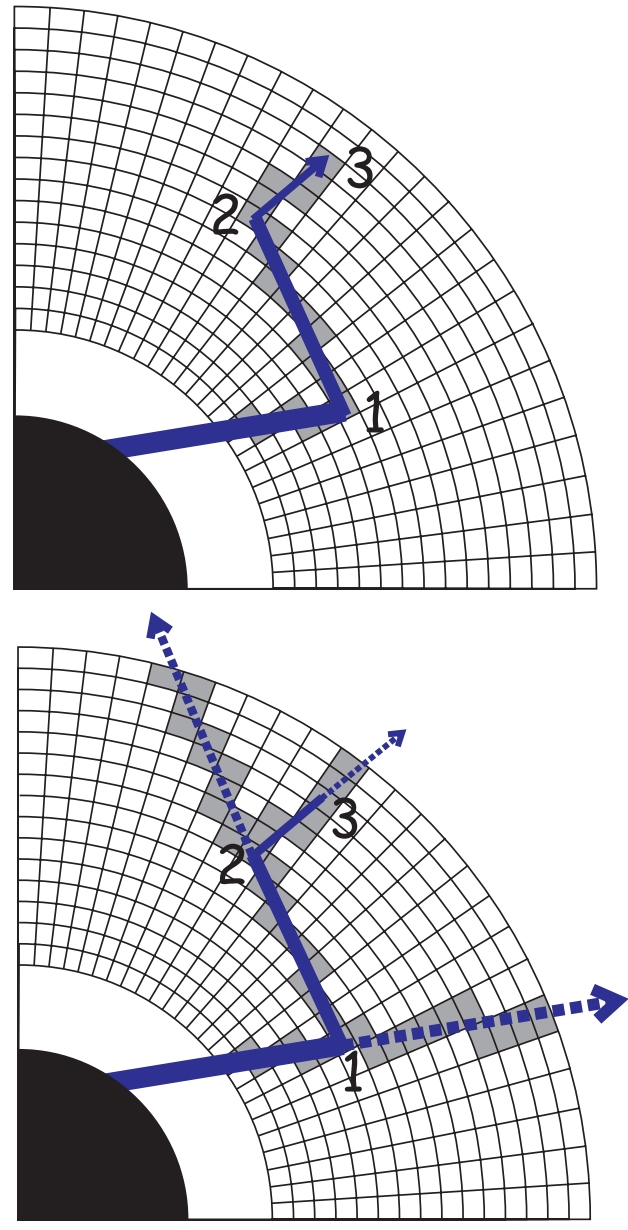

Fig. 2. Propagation routines. In our method 1 (upper figure), additional information about the mean intensity is gained along the photon trajectories between the interaction points 1,2,3. The lower figure depicts our method 2, where the absorption along the trajectories is treated in a deterministic way. Photon packages can be emitted from the star (as depicted) or from any cell.

$j_{\theta}$ is the index of the escape latitude angle interval defined by $\theta_{j}^{\text {esc }}<\theta^{\text {esc }}<\theta_{j+1}^{\text {esc }}$. In this case, the propagation is finished.

The propagation also stops if the PP hits the star. In that case, $\Gamma_{\lambda}\left(x^{\prime}, \star\right)$, the energy gain of the star due to photons emitted by $x^{\prime}$, is incremented as

$$
\Gamma_{\lambda}\left(x^{\prime}, \star\right) \leftarrow \Gamma_{\lambda}\left(x^{\prime}, \star\right)+p_{\lambda} .
$$

In all other cases, the next interaction point $\boldsymbol{r}$ associated with $\tau_{\lambda}$ lies inside the model volume and is obtained by linear interpolation between the pre-calculated $\tau_{\lambda}(s)$-points.

$\boldsymbol{r}=\boldsymbol{r}_{j}+\left(\boldsymbol{r}_{j+1}-\boldsymbol{r}_{j}\right) \frac{\tau_{\lambda}-\tau_{\lambda}^{j}}{\tau_{\lambda}^{j+1}-\tau_{\lambda}^{j}}$

$j$ is given by the constraint $\tau_{\lambda}{ }^{j}<\tau_{\lambda}<\tau_{\lambda}{ }^{j+1}$. At the interaction point $\boldsymbol{r}$ located in cell $\xi$, the photon package is partly absorbed and partly scattered in a new direction. This splitting of energy is treated in a deterministic way according to the albedo $\gamma_{\lambda}^{\xi}$. The gain of energy by cell $\xi$ via absorption of photons emitted from $x^{\prime}, \Gamma_{\lambda}\left(x^{\prime}, \xi\right)$, is incremented as

$\Gamma_{\lambda}\left(x^{\prime}, \xi\right) \leftarrow \Gamma_{\lambda}\left(x^{\prime}, \xi\right)+p_{\lambda}\left(1-\gamma_{\lambda}^{\xi}\right)$.
The remaining fraction of the photon package is scattered in a new direction, which results from the local phase function $g_{\lambda}^{\xi}$ according to a probabilistic treatment of the scattering angle as described in Appendix B

$\boldsymbol{r}_{0} \leftarrow \boldsymbol{r}$
$\widehat{\boldsymbol{n}} \leftarrow f\left(g_{\lambda}^{\xi}, \widehat{\boldsymbol{n}}\right)$
$p_{\lambda} \leftarrow p_{\lambda} \gamma_{\lambda}^{\xi}$.

The propagation procedure is continued with the reduced $p_{\lambda}$ into the new direction $\widehat{\boldsymbol{n}}$, until the photon package has left the model volume, has hit the star or if its energy has become vanishingly small, e.g. $p_{\lambda}<10^{-6} p_{\lambda}^{0}$.

After all stellar and cell photon packages have been propagated, the mean intensity $J_{\lambda}$ in cell $\xi$ can be calculated according to Lucy (1999) by

$J_{\lambda}(\xi)=\frac{1}{4 \pi V(\xi)} \sum_{x^{\prime}} j_{\lambda}\left(x^{\prime}, \xi\right)$.

The observable flux $F_{\lambda}\left(j_{\theta}\right)$ within the latitude angular interval of the escape direction $j_{\theta}$ at distance $d$ towards the observer is calculated by

$F_{\lambda}\left(j_{\theta}\right)=\frac{1}{d^{2}} \frac{1}{\Omega\left(j_{\theta}\right)} \sum_{x^{\prime}} f_{\lambda}\left(x^{\prime}, j_{\theta}\right)$,

where $d^{2} \Omega\left(j_{\theta}\right)=2 \pi\left(\cos \theta_{j}^{\text {esc }}-\cos \theta_{j+1}^{\text {esc }}\right)$ is the area of the $j_{\theta}$ 's interval at distance $d$.

\subsubsection{Method 2}

Within the scope of this method, the absorption and extinction of the photon package along the complete ray (up to the final optical depth integration point $\tau_{\lambda}{ }^{n}$ ) is treated in a deterministic way, whereas the scattering is accounted for by choosing one particular scattering point along the ray in a probabilistic way.

Considering the absorption, we directly use the results of the optical depth integration in order to analytically determine the energy gains of the crossed cells by absorption. The energy gains of all cells $\xi$ encountered by the ray, even beyond the chosen scattering point (see lower diagram of Fig. 2) are incremented according to

$\Gamma_{\lambda}\left(x^{\prime}, \xi\right) \leftarrow \Gamma_{\lambda}\left(x^{\prime}, \xi\right)+p_{\lambda} \alpha_{\mathrm{abs}}^{\xi}$

where $\alpha_{\mathrm{abs}}^{\xi}$ is the fraction of $p_{\lambda}$ absorbed by cell $\xi$. Defining $j_{\xi}^{\text {in }}$ and $j_{\xi}^{\text {out }}$ as the indices of the optical depth points located at the entrance and the exit of cell $\xi$, respectively, and using the assumption that the albedo $\gamma_{\lambda}^{\xi}$ is constant in each cell, $\alpha_{\text {abs }}^{\xi}$ is given by

$\alpha_{\mathrm{abs}}^{\xi}=\left(1-\gamma_{\lambda}^{\xi}\right)\left(\mathrm{e}^{-\tau_{\lambda}^{j_{\xi}^{\text {in }}}}-\mathrm{e}^{-\tau_{\lambda}^{j_{\xi}^{\text {out }}}}\right)$

Another fraction $\alpha_{\text {esc }}$ of the photon package leaves the model volume and hence contributes to the observable flux in escape direction $\widehat{n}$

$f_{\lambda}\left(x^{\prime}, j_{\theta}\right) \leftarrow f_{\lambda}\left(x^{\prime}, j_{\theta}\right)+p_{\lambda} \alpha_{\mathrm{esc}}$, 
or, if $\tau_{\lambda}^{n}$ lies onto the stellar surface, hits the star

$\Gamma_{\lambda}\left(x^{\prime}, \star\right) \leftarrow \Gamma_{\lambda}\left(x^{\prime}, \star\right)+p_{\lambda} \alpha_{\mathrm{esc}}$.

The escaping fraction of the photon package is given by

$\alpha_{\text {esc }}=\mathrm{e}^{-\tau_{\lambda}^{n}}$.

The total scattered fraction $\alpha_{\text {sca }}$ of the photon package can finally be obtained by considering the energy conservation $\sum_{\xi} \alpha_{\mathrm{abs}}^{\xi}+\alpha_{\mathrm{sca}}+\alpha_{\mathrm{esc}}=1$, and by using Eqs. (29) and (32)

$\alpha_{\text {sca }}=\sum_{\xi} \gamma_{\lambda}^{\xi}\left(\mathrm{e}^{-\tau_{\lambda} j_{\xi}^{\text {in }}}-\mathrm{e}^{-\tau_{\lambda}{ }^{j_{\xi} \text { out }}}\right)$.

The choice of the optical depth of the scattering point $\tau_{\lambda}$ is done noting that the probability to be scattered at $\tau_{\lambda}$ is the product of:

1. the probability $P_{\xi}(\xi)$ that the scattering point lies inside the cell $\xi$

$P_{\xi}(\xi)=\int_{\tau_{\lambda}}^{\tau_{\lambda} \tau_{\xi}^{j_{\xi} \text { out }}} P_{\tau_{\lambda}}\left(\tau_{\lambda}\right) \mathrm{d} \tau_{\lambda}=\frac{\gamma_{\lambda}^{\xi}}{\alpha_{\text {sca }}}\left(\mathrm{e}^{-\tau_{\lambda}^{j_{\xi}^{\text {in }}}}-\mathrm{e}^{-\tau_{\lambda}^{j_{\xi}^{\text {out }}}}\right) ;$

2. the probability $P_{\tau_{\lambda}}\left(\tau_{\lambda} \mid \xi\right) d \tau_{\lambda}$ that the scattering point lies between $\tau_{\lambda}$ and $\tau_{\lambda}+d \tau_{\lambda}$, once we know that it is located in $\xi$

$P_{\tau_{\lambda}}\left(\tau_{\lambda} \mid \xi\right)=\frac{\mathrm{e}^{-\tau_{\lambda}}}{\mathrm{e}^{-\tau_{\lambda}{ }^{{ }^{\mathrm{in}}}-\mathrm{e}^{-\tau_{\lambda}{ }^{{ }^{\mathrm{j}}{ }_{\xi}^{\text {ut }}}}}}$.

For the choice of the scattering point $\boldsymbol{r}$ we therefore proceed in three steps. We first select the cell $\xi$ that contains the scattering point $\boldsymbol{r}$ according to Eq. (34) by means of an equally distributed random number $z_{1} \in[0,1)$.

Second, we choose the optical depth of the scattering point in cell $\xi$ according to Eq. (35) by means of another random number $z_{2} \in[0,1)$ with

$\tau_{\lambda}=-\ln \left[\mathrm{e}^{-\tau_{\lambda}^{j \text { in }}}-z_{2}\left(\mathrm{e}^{-\tau_{\lambda}^{j \text { in }}}-\mathrm{e}^{-\tau_{\lambda}^{j \text { out }}}\right)\right]$.

Third, we determine the location of the scattering point $\boldsymbol{r}$ which corresponds to $\tau_{\lambda}$.

The subsequent considerations of method 2 are similar to those of method 1 concerning the scattered fraction of the photon package into a new propagation direction

$$
\begin{aligned}
& \boldsymbol{r}_{0} \leftarrow \boldsymbol{r} \\
& \widehat{\boldsymbol{n}} \leftarrow f\left(g_{\lambda}^{\xi}, \widehat{\boldsymbol{n}}\right) \\
& p_{\lambda} \leftarrow p_{\lambda} \alpha_{\text {sca }} .
\end{aligned}
$$

The propagation procedure is repeated with the reduced $p_{\lambda}$ into the new direction $\widehat{\boldsymbol{n}}$, until the photon package luminosity has become vanishingly small.

\section{Temperature determination}

The temperature structure of the illuminated matter is determined by assuming LTE and radiative equilibrium at every point $\boldsymbol{r}$ in the model volume. In the general case, where the opacities $\kappa_{\lambda}^{\text {abs }}, \kappa_{\lambda}^{\text {sca }}$ and $g_{\lambda}$ depend explicitly on the temperature of the medium, the consistent solution of the radiative transfer problem requires an iteration between the calculation of the radiative transfer (together with the auxiliary constraint of radiative equilibrium) and the calculation of the opacities. In consideration of Monte Carlo methods, this means that, in general, the creation and propagation of photon packages must be performed several times, until convergence with the opacity and temperature structure is achieved (e.g. Lucy et al. 1999).

However, this time-consuming iteration can be avoided if the opacities $\kappa_{\lambda}^{\mathrm{abs}}$ and $\kappa_{\lambda}^{\mathrm{sca}}$ are explicitly temperatureindependent. Applying Mie-theory, the opacities of dusty media depend only on the size and the optical properties of the dust grain material but do not depend explicitly on the temperature. In this special case, it is possible to determine the proper temperature structure after the propagation of all photon packages has been completed.

The basic idea in this case is as follows. The probabilities for all physical events to occur during the photon propagation (absorption, angle-dependent scattering) are fully determined by the opacities along the ray. Consequently, if the opacities are fixed and two Monte Carlo experiments $\mathcal{A}$ and $\mathcal{B}$ with different temperature structures, $\left\{T_{\star}^{\mathcal{A}}, T_{\xi^{\prime}}^{\mathcal{A}}\right\}$ and $\left\{T_{\star}^{\mathcal{B}}, T_{\xi^{\prime}}^{\mathcal{B}}\right\}$ are considered all the primary information produced by the Monte Carlo experiment $\mathcal{F}_{\lambda}$ remains the same except for a correction factor that scales the initial photon package energies $p_{\lambda}^{0}$

$\mathcal{F}_{\lambda}^{\mathcal{B}}=\mathcal{F}_{\lambda}^{\mathcal{A}} \frac{B_{\lambda}\left(T_{x^{\prime}}^{\mathcal{B}}\right)}{B_{\lambda}\left(T_{x^{\prime}}^{\mathcal{A}}\right)}$,

where $\mathcal{F}_{\lambda}$ can be one of the following quantities: $\Gamma_{\lambda}, j_{\lambda}$ or $f_{\lambda}$. The source function $B_{\lambda}\left(T_{x^{\prime}}\right)$ refers to the emitter of the photon package, i.e. $T_{x^{\prime}}=T_{\star}$ for stellar photons and $T_{x^{\prime}}=T_{\xi^{\prime}}$ for photons emitted by cell $\xi^{\prime}$.

\subsection{Method 1}

In this method, we use the formulation of the radiative equilibrium in LTE by means of the mean intensity $J_{\lambda}$ (Eq. (3)). The determination of $J_{\lambda}$ by a Monte Carlo experiment is necessarily restricted to an average sense, namely to a mean value over the volume of the considered cell $\xi$. Consequently, the mean intensity is assumed to be constant within one cell $J_{\lambda}(\boldsymbol{r})=J_{\lambda}(\xi)$ for $\boldsymbol{r} \in V(\xi)$. Equation (3) then results in

$\sum_{i=1}^{N_{\lambda}} w_{i} \hat{\kappa}_{\lambda_{i}}^{\mathrm{abs}}(\xi) B_{\lambda_{i}}\left(T_{\xi}\right)=\sum_{i=1}^{N_{\lambda}} w_{i} \hat{\kappa}_{\lambda_{i}}^{\mathrm{abs}}(\xi) J_{\lambda_{i}}(\xi)$,

where we have approximated the $\lambda$-integrals by a sum over wavelengths points $\lambda_{i}\left(i=1, \ldots, N_{\lambda}\right)$ with general $\lambda$-integration weights $w_{i}$, applying a second order discretisation scheme

$\int_{\lambda_{1}}^{\lambda_{N_{\lambda}}} f(\lambda) \mathrm{d} \lambda \approx \sum_{i=1}^{N_{\lambda}} w_{i} f\left(\lambda_{i}\right)$

By means of Eq. (38), the mean intensity according to Lucy (1999) is given by

$J_{\lambda_{i}}(\xi)=\frac{1}{4 \pi V(\xi)} \sum_{x^{\prime}} \frac{j_{\lambda_{i}}\left(x^{\prime}, \xi\right)}{B_{\lambda_{i}}\left(T_{x^{\prime}}^{0}\right)} B_{\lambda_{i}}\left(T_{x^{\prime}}\right)$ 
where $T_{x^{\prime}}^{0}$ are initial guesses of the stellar and the cell temperatures, respectively, the primary Monte Carlo information $j_{\lambda}\left(x^{\prime}, \xi\right)$ was computed from. Equation (41) has the formal structure of the well-known $\Lambda$-operator

$$
\begin{aligned}
& J_{\lambda_{i}}(\xi)=\sum_{x^{\prime}} \Lambda_{\xi, x^{\prime}}^{i} B_{\lambda_{i}}\left(T_{x^{\prime}}\right) \\
& \Lambda_{\xi, x^{\prime}}^{i}=\frac{1}{4 \pi V(\xi)} \frac{j_{\lambda_{i}}\left(x^{\prime}, \xi\right)}{B_{\lambda_{i}}\left(T_{x^{\prime}}^{0}\right)} .
\end{aligned}
$$

For temperature-independent opacities, the $\Lambda$-operator is simply a matrix with constant coefficients, which is filled by the Monte Carlo experiment. By combining Eq. (39) with Eq. (42) we obtain

$$
\sum_{i=1}^{N_{\lambda}} w_{i} \hat{\kappa}_{\lambda_{i}}^{\mathrm{abs}}(\xi) B_{\lambda_{i}}\left(T_{\xi}\right)=\sum_{i=1}^{N_{\lambda}} w_{i} \hat{\kappa}_{\lambda_{i}}^{\mathrm{abs}}(\xi) \sum_{x^{\prime}} \Lambda_{\xi, x^{\prime}}^{i} B_{\lambda_{i}}\left(T_{x^{\prime}}\right)
$$

Equation (44) forms a system of $\left(N_{r} \cdot N_{\theta}\right)$ nonlinear equations for the $\left(N_{r} \cdot N_{\theta}+1\right)$ unknown temperatures $\left\{T_{\star}, T_{\xi}\right\}$. The one missing equation is given by the specification of the total flux through the stellar surface by means of the effective temperature $T_{\text {eff }}$

$4 \pi R_{\star}^{2} \sigma T_{\text {eff }}^{4}=4 \pi R_{\star}^{2} \int_{0}^{\infty} F_{\lambda}^{+}\left(R_{\star}\right)-F_{\lambda}^{-}\left(R_{\star}\right) \mathrm{d} \lambda$.

$F_{\lambda}^{+}\left(R_{\star}\right)$ is the outgoing flux, specified by $T_{\star}$, and $F_{\lambda}{ }^{-}\left(R_{\star}\right)$ is the incoming flux, consisting of photon packages which are scattered back or are thermally re-emitted from the dust shell and hit the star

$4 \pi R_{\star}^{2} \sigma T_{\mathrm{eff}}^{4}=4 \pi R_{\star}^{2} \sigma T_{\star}^{4}-\sum_{i=1}^{N_{\lambda}} w_{i} \sum_{x^{\prime}} \frac{\Gamma_{\lambda_{i}}\left(x^{\prime}, \star\right)}{B_{\lambda_{i}}\left(T_{x^{\prime}}^{0}\right)} B_{\lambda_{i}}\left(T_{x^{\prime}}\right)$.

According to Eq. (46) we find $T_{\star} \geq T_{\text {eff }}$, which expresses the additional radiative heating of the stellar atmosphere due to the presence of a circumstellar environment.

Equations (44) and (46) form a complete system of nonlinear equations for the unknown temperatures which can be solved by iterative methods as will be explained in Sect. 4.3.

\subsection{Method 2}

In this method, the radiative equilibrium is formulated in terms of the equality of the total emission and the total absorption of radiative energy per time, integrated over the volume of cell $\xi$

$\int_{0}^{\infty} \int_{\mathrm{V}(\xi)} 4 \pi \kappa_{\lambda}^{\mathrm{abs}}(\boldsymbol{r}) B_{\lambda}(T(\boldsymbol{r})) \mathrm{d}^{3} r \mathrm{~d} \lambda=\int_{0}^{\infty} \mathcal{A}_{\lambda}(\xi) \mathrm{d} \lambda$.

$\mathcal{A}_{\lambda}(\xi)$ is the amount of absorbed energy by cell $\xi$ per time and unit wavelength, which results from the absorption events in the Monte Carlo experiment. Using Eq. (38), $\mathcal{A}_{\lambda}(\xi)$ can be expressed by

$$
\mathcal{A}_{\lambda}(\xi)=\sum_{x^{\prime}} \frac{\Gamma_{\lambda}\left(x^{\prime}, \xi\right)}{B_{\lambda}\left(T_{x^{\prime}}^{0}\right)} B_{\lambda}\left(T_{x^{\prime}}\right),
$$

where again $T_{x^{\prime}}^{0}$ are initial guesses for the stellar and cell temperatures, respectively, the primary Monte Carlo information $\Gamma_{\lambda}\left(x^{\prime}, \xi\right)$ was computed from. Equation (47) can be re-written as

$4 \pi M_{\xi} \int_{0}^{\infty} \hat{\kappa}_{\lambda}^{\mathrm{abs}}(\xi) B_{\lambda}\left(T_{\xi}\right) \mathrm{d} \lambda=\int_{0}^{\infty} \mathcal{A}_{\lambda}(\xi) \mathrm{d} \lambda$,

where $M_{\xi}=\int_{\mathrm{V}(\xi)} \rho(\boldsymbol{r}) \mathrm{d}^{3} r$ is the mass of cell $\xi$. Using $\lambda$-integration weights, Eq. (49) results in

$4 \pi M_{\xi} \sum_{i=1}^{N_{\lambda}} w_{i} \hat{\kappa}_{\lambda_{i}}^{\mathrm{abs}}(\xi) B_{\lambda_{i}}\left(T_{\xi}\right)=\sum_{i=1}^{N_{\lambda}} w_{i} \mathcal{A}_{\lambda_{i}}(\xi)$

We can combine Eqs. (50) and (48) to get the following equation

$$
\sum_{i=1}^{N_{\lambda}} w_{i} \hat{\kappa}_{\lambda_{i}}^{\mathrm{abs}}(\xi) B_{\lambda_{i}}\left(T_{\xi}\right)=\sum_{i=1}^{N_{\lambda}} w_{i} \sum_{x^{\prime}} \Delta_{\xi, x^{\prime}}^{i} B_{\lambda_{i}}\left(T_{x^{\prime}}\right)
$$

where the operator $\Delta_{\xi, x^{\prime}}^{i}$ is defined as

$\Delta_{\xi, x^{\prime}}^{i}=\frac{1}{4 \pi M_{\xi}} \frac{\Gamma_{\lambda_{i}}\left(x^{\prime}, \xi\right)}{B_{\lambda_{i}}\left(T_{x^{\prime}}^{0}\right)}$.

Equation (51) together with Eq. (46) form a complete system of $\left(N_{r} \cdot N_{\theta}+1\right)$ nonlinear equations for the $\left(N_{r} \cdot N_{\theta}+1\right)$ unknown temperatures $\left\{T_{\star}, T_{\xi}\right\}$.

\subsection{Solution of the equation system}

The system of non-linear equations (44)/(51) and (46) for method $1 / 2$, respectively, can be solved by standard numerical methods, e.g. by means of a Newton-Raphson iteration ${ }^{6}$.

Two problems may be encountered during this iteration. First, the numerical inversion of the full Jacobi matrix with dimension $\left(N_{r} \cdot N_{\theta}+1\right)^{2}$ can become too time-expensive for large grids or may require too much computer memory. Second, the convergence of the Newton-Raphson iteration may fail, if the initial guesses $\left\{T_{\star}^{0}, T_{\xi}^{0}\right\}$ are too far away from the actual solution.

An alternative method is a simple $\Lambda$-type iteration. This method is faster for large grids and can be used as preiteration to provide better initial values for the NewtonRaphson method. In the $\Lambda$-iteration technique, the equations to be solved are interpreted as a direct rule for one iteration step $\left\{T_{\star}^{(n-1)}, T_{\xi}^{(n-1)}\right\} \rightarrow\left\{T_{\star}^{(n)}, T_{\xi}^{(n)}\right\}$. The stellar temperature $T_{\star}$ is iterated as

$$
\begin{aligned}
4 \pi R_{\star}^{2} \sigma\left(T_{\star}^{(n)}\right)^{4}= & 4 \pi R_{\star}^{2} \sigma T_{\text {eff }}^{4} \\
& +\sum_{i=1}^{N_{\lambda}} w_{i} \sum_{x^{\prime}} \frac{\Gamma_{\lambda_{i}}\left(x^{\prime}, \star\right)}{B_{\lambda_{i}}\left(T_{x^{\prime}}^{0}\right)} B_{\lambda_{i}}\left(T_{x^{\prime}}^{(n-1)}\right) .
\end{aligned}
$$

\footnotetext{
${ }^{6}$ We use the routine MnEwT from the Numerical Recipes (1986).
} 
In the framework of method 1 , the cell temperatures are iterated as

$$
\begin{aligned}
& \sum_{i=1}^{N_{\lambda}} w_{i} \hat{\kappa}_{\lambda_{i}}^{\mathrm{abs}}(\xi)\left(1-\Lambda_{\xi, \xi}^{i}\right) B_{\lambda_{i}}\left(T_{\xi}^{(n)}\right)= \\
& \sum_{i=1}^{N_{\lambda}} w_{i} \hat{\kappa}_{\lambda_{i}}^{\mathrm{abs}}(\xi) \sum_{x^{\prime} \neq \xi} \Lambda_{\xi, x^{\prime}}^{i} B_{\lambda_{i}}\left(T_{x^{\prime}}^{(n-1)}\right),
\end{aligned}
$$

whereas in the framework of method 2

$$
\begin{aligned}
& \sum_{i=1}^{N_{\lambda}} w_{i}\left(\hat{\kappa}_{\lambda_{i}}^{\mathrm{abs}}(\xi)-\Delta_{\xi, \xi}^{i}\right) B_{\lambda_{i}}\left(T_{\xi}^{(n)}\right)= \\
& \sum_{i=1}^{N_{\lambda}} w_{i} \sum_{x^{\prime} \neq \xi} \Delta_{\xi, x^{\prime}}^{i} B_{\lambda_{i}}\left(T_{x^{\prime}}^{(n-1)}\right) .
\end{aligned}
$$

The convergence of the applied method and the quality of the solution can be checked by controlling the condition of global radiative equilibrium. This equilibrium can be expressed in terms of the equality of the energy per time totally absorbed by the dust shell and the total luminosity emitted by and escaping from the dust shell, either through the inner boundary (star hits) or through the outer boundary (observable flux)

$$
\begin{aligned}
\sum_{i=1}^{N_{\lambda}} w_{i} \sum_{\xi} \frac{\Gamma_{\lambda}(\star, \xi)}{B_{\lambda}\left(T_{\star}^{0}\right)} B_{\lambda}\left(T_{\star}\right)= \\
\sum_{i=1}^{N_{\lambda}} w_{i} \sum_{\xi}\left(\Gamma_{\lambda_{i}}(\xi, \star)+\sum_{j_{\theta}} f_{\lambda_{i}}\left(\xi, j_{\theta}\right)\right) \frac{B_{\lambda_{i}}\left(T_{\xi}\right)}{B_{\lambda_{i}}\left(T_{\xi}^{0}\right)} .
\end{aligned}
$$

The well-known drawback of the $\Lambda$-iteration scheme is its slow convergence in case of large optical depths. One way to accelerate this convergence is to occasionally perform one iteration step as $\left\{T_{\xi}^{(n)}\right\}=\left\{\alpha T_{\xi}^{(n-1)}\right\}$ where the common factor $\alpha$ is chosen such that Eq. (56) becomes temporarily true, and then to return to the ordinary iteration scheme according to Eqs. (53) and (54)/(55).

The iteration is carried on until the relative difference between the 1.h.s. and the r.h.s. of the global condition of radiative equilibrium (Eq. (56)) becomes smaller than a given value. Finally, for not too large grids, the $\Lambda$-iteration can be completed by a few Newton-Raphson iteration steps.

Concerning method 2, this iteration scheme is found to be able to find the simultaneous solution of Eqs. (46), (51) and (56) with any prescribed precision down to the machine level. Concerning method 1, Eqs. (44) and (46) can be solved simultaneously with any prescribed precision, but there is usually a small remaining error in Eq. (56), being typically less than one percent. This error arrises from the fact that in method 1 , the local condition of radiative equilibrium is calculated via the determination of mean intensities (Eq. (44)) whereas the constraint of global radiative equilibrium is checked via absorbed energies. Since $j_{\lambda}$ and $\Gamma_{\lambda}$ are determined independently, they are subject to an independent Monte Carlo noise and cannot be expected to produce simultaneous solutions.

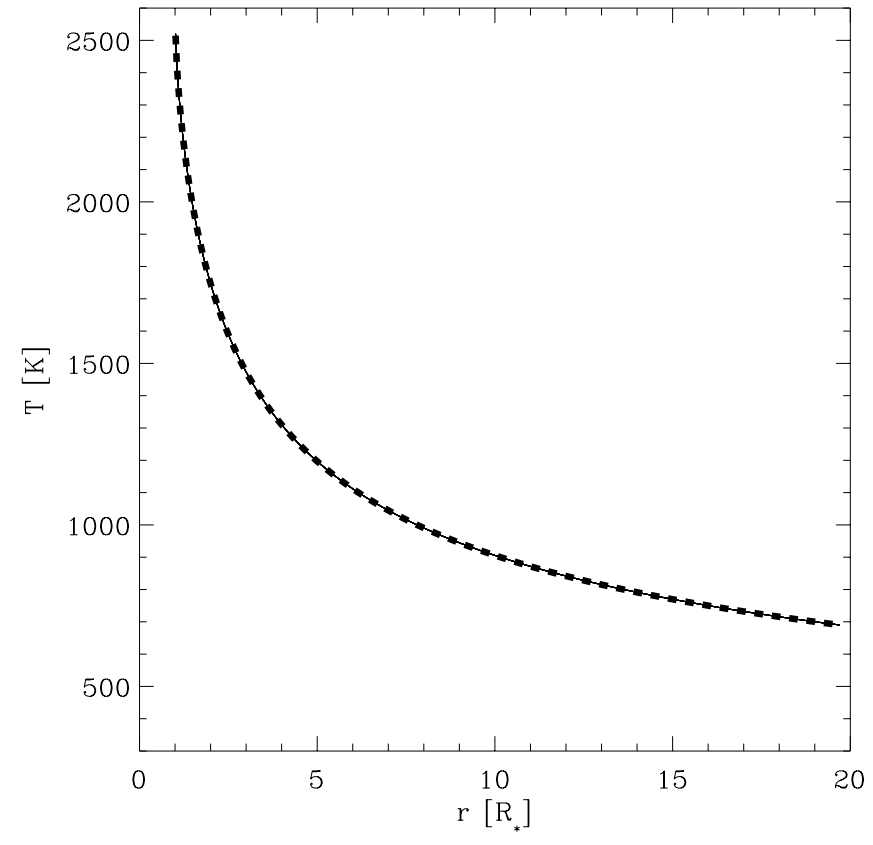

Fig. 3. Optically thin case with $T_{\text {eff }}=T_{\star}=3000 \mathrm{~K}$ and $\kappa_{\lambda}^{\text {abs }} \propto 1 / \lambda$. The analytical reference solution $T_{\text {ref }}(r)$ (Eq. (60) with $p=1$ ) is shown as thick dashed line. The 2D results obtained with the Monte Carlo method $T(r, \theta)$ are depicted as 10 solid lines connecting the points along constant $\theta$. Because of the small Monte Carlo noise, these lines appear as a single thin line almost exactly fitting the reference solution.

\section{1D-test calculations}

\subsection{The optically thin case}

In the optically thin limiting case, the radiation field around a black body sphere of radius $R_{\star}$ and temperature $T_{\star}$ is given by

$J_{\lambda}(\boldsymbol{r})=W(r) B_{\lambda}\left(T_{\star}\right)$

$W(r)=\frac{1}{2}\left(1-\sqrt{1-\frac{R_{\star}^{2}}{r^{2}}}\right)$,

where $W(r)$ is the radial dilution factor. For a power-lawdependence of the absorption coefficient

$\kappa_{\lambda}^{\mathrm{abs}} \propto \lambda^{-p}$

the radiative equilibrium (Eq. (3)) can be solved analytically, resulting in a reference solution

$T_{\text {ref }}(\boldsymbol{r})=(W(r))^{\frac{1}{4+p}} T_{\star}$

We take this analytical solution as a first test of our Monte Carlo code. We choose $T_{\star}=3000 \mathrm{~K}$ and $p=1$. The following spatial and wavelength grids are considered: $N_{\lambda}=40$ logarithmically equidistant wavelength grid points between $0.1 \mu \mathrm{m}$ and $100 \mu \mathrm{m}, N_{r}=100$ logarithmically equidistant radial grid points between $1 R_{\star}$ and $20 R_{\star}, N_{\theta}=10$ equidistant latitude angular grid points between 0 and $\pi$. A total number of $8 \times 10^{6}$ stellar photon packages is used. 
In spherical symmetry, the Monte Carlo errors can be expressed by the standard deviations of the resulting temperatures at constant radii

$$
\begin{aligned}
& \langle T\rangle_{\theta}\left(r_{i}\right)=\frac{1}{N_{\theta}} \sum_{k=1}^{N_{\theta}} T\left(r_{i}, \theta_{k}\right) \\
& \sigma_{T}\left(r_{i}\right)^{2}=\frac{1}{N_{\theta}-1} \sum_{k=1}^{N_{\theta}}\left(T\left(r_{i}, \theta_{k}\right)-\langle T\rangle_{\theta}\left(r_{i}\right)\right)^{2} .
\end{aligned}
$$

Figure 3 shows the excellent agreement between the results of the Monte Carlo method and the reference solution. The temperature noise $( \pm 0.03 \mathrm{~K}$ on average) is very small all over the model volume. The relative difference between the reference solution and $\langle T\rangle_{\theta}$ is at most $0.2 \%$. This relatively large error occurs in the innermost cells $\left(r / R_{\star} \in[1,1.0304]\right)$, demonstrating the trouble with the proper definition of mean values in extended cells, when the temperature gradient is very steep $\left(T_{\text {ref }}\left(R_{\star}\right)=2611.65 \mathrm{~K}\right.$ and $\left.T_{\text {ref }}\left(1.0304 R_{\star}\right)=2471.42 \mathrm{~K}\right)$. For the remainder of the cells, the mean relative difference is quite small $0.002 \%$.

This test calculation indicates that the problems of the standard Monte Carlo technique at small optical depths can be convincingly solved by the determination of mean intensities, as proposed by Lucy (1999).

\subsection{An optically thick spherical envelope}

As our second test problem, we consider a spherically symmetric, optically thick dust envelope around a black body star. We use the artificial absorption coefficient specified by Eq. (59) and put $\kappa_{\lambda}^{\mathrm{sca}}(\boldsymbol{r})=\kappa_{\lambda}^{\text {abs }}(\boldsymbol{r})$, i.e. $\gamma_{\lambda}(\boldsymbol{r})=1 / 2$. For the mass density, a $1 / r^{2}$-law is used. We assume isotropic scattering $g_{\lambda}(\boldsymbol{r}, \vartheta)=1$. The inner boundary of the dust envelope is located at $r=3 R_{\star}$. In the inner cavity $r / R_{\star} \in[1,3]$ the opacities are set to zero ${ }^{7}$. The opacity is scaled by specifying the total radial optical depth through the dust envelope to a given value at a given wavelength, here $\tau_{1 \mu \mathrm{m}}=10$.

A reference solution $T_{\text {ref }}(r)$ was kindly provided by T. Le Bertre who solved this test problem by means of a deterministic code developed by Leung (1976), assuming spherical symmetry. In this code, the stellar temperature $T_{\star}$ is specified rather than the effective temperature $T_{\text {eff }}$. Therefore, we consider $T_{\star}=2500 \mathrm{~K}$ as fixed for this test and solve Eq. (46) for $T_{\text {eff }}$ instead. The difference is quite substantial: about $15 \%$ of the irradiated stellar luminosity returns to the star in this particular case.

Again, $N_{\lambda}=40$ logarithmically equidistant wavelength grid points between $0.1 \mu \mathrm{m}$ and $100 \mu \mathrm{m}$ are used. The spatial grid is adapted to the reference solution in such a way, that the radial cell centre positions coincide with the given 65 points between $3 R_{\star}$ and $300 R_{\star} .20$ additional radial grid points are included in order to sample the inner (dust-free) cavity from $1 R_{\star}$ to $3 R_{\star}$ which has no influence on the temperature distribution

\footnotetext{
7 Completely empty cells cannot be handled by method 2 . In order to avoid these problems, we add a tiny background opacity for method 2 models, which has the same spectral characteristic and only changes the optical depths by a factor of less than $10^{-8}$.
}

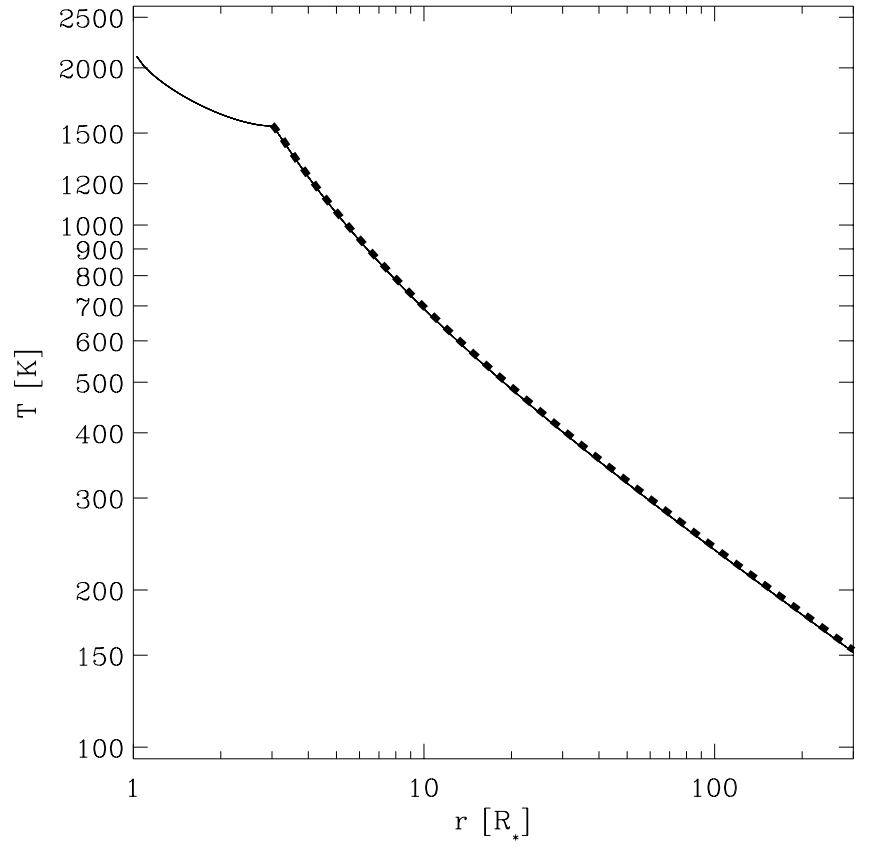

Fig. 4. Optically thick envelope $\left(\tau_{1 \mu \mathrm{m}}=10\right)$ between $3 R_{\star}$ and $300 R_{\star}$ with $1 / \lambda$-opacities (see text). The reference solution $T_{\text {ref }}(r)$ is shown as thick dashed line. The $2 \mathrm{D}$ results obtained with the Monte Carlo method $T(r, \theta)$ are depicted by 10 thin solid lines connecting the resultant temperatures along constant $\theta$. All results accurately fit the reference solution. The stellar temperature has been fixed for this calculation $T_{\star}=2500 \mathrm{~K}$, whereas the effective temperature results to be $T_{\text {eff }}=2454 \mathrm{~K}$.

further out in the dust envelope. $N_{\theta}=10$ equidistant latitude angular grid points between 0 and $\pi$ are considered.

We have performed two Monte Carlo calculations for this test problem, using method 1 and method 2 . For method 1 , we have used $8 \times 10^{6}$ stellar and $8 \times 10^{6}$ cell photon packages. For method 2 , we have used only $1.2 \times 10^{6}$ stellar and $1.2 \times 10^{6}$ cell photon packages. This is because method 2 is more timeconsuming, since the deterministic treatment of the absorption requires the complete propagation of the photons up to the end of each ray and involves the calculation of many exponential functions. The number of photon packages for the method 2-model was chosen such that the overall computing time (about 500 s on a Cray T3E-1200 parallel computer using 16 processors) was finally similar to the method 1 model.

Figure 4 depicts the results of the method 1 model in comparison to the reference solution. Figure 5 shows the systematic deviations for both methods. Again, the Monte Carlo results agree well with the reference solution. There are some slight systematic deviations as $T(\operatorname{method} 1)<T(\operatorname{method} 2)<$ $T_{\text {ref }}$, but these deviations are everywhere smaller than $10 \mathrm{~K}$ $(<1 \%)^{8}$. The noise level in the temperature determination of

\footnotetext{
${ }^{8}$ Since the deterministic reference code uses a $\Lambda$-type temperature iteration scheme, the convergence of the Leung-code is already quite slow at $\tau_{1 \mu \mathrm{m}}=10$. Although T. Le Bertre has performed more than 50 iteration steps (much more than what was usually considered as necessary), there were still slightly increasing temperatures to be observed at the end of the iteration. Due to this behaviour, we are not completely sure that the "reference solution" is actually more precise than our Monte Carlo solutions.
} 


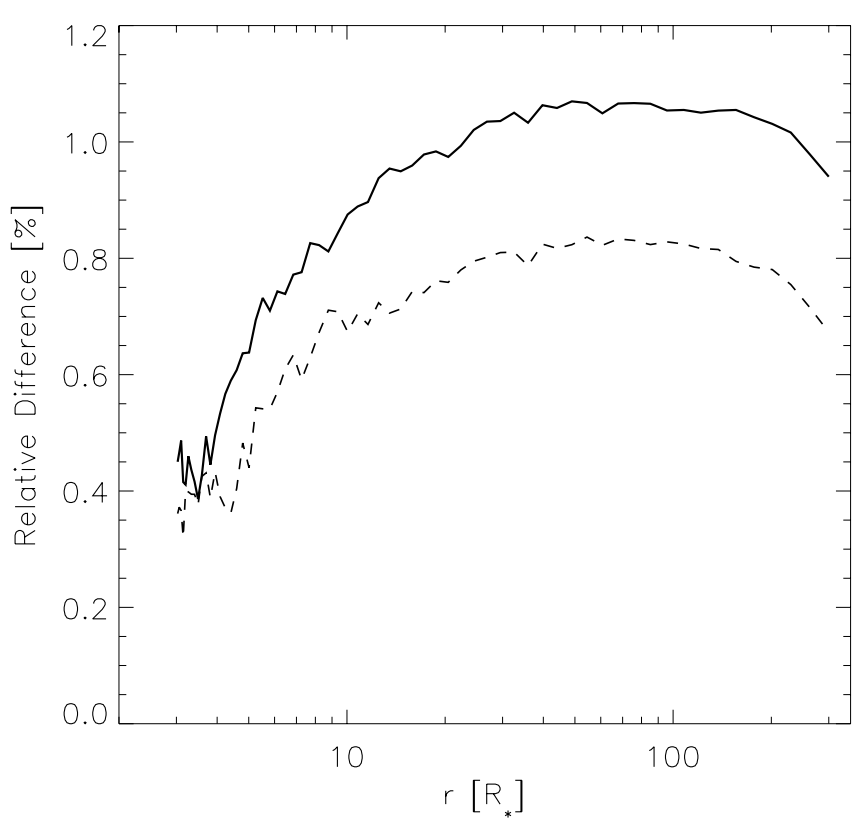

Fig. 5. Relative deviations between the Monte Carlo results and the reference solution $\left(T_{\text {ref }}(r)-\langle T\rangle_{\theta}(r)\right) / T_{\text {ref }}(r)$ for the optically thick dust envelope as shown in Fig. 4. The results of method 1 and 2 are depicted as full and dashed lines, respectively. All deviations are smaller than $1.1 \%$ and $0.8 \%$ for method 1 and 2 , respectively.

our Monte Carlo codes is small: method 1 achieves a slightly lower mean standard deviation $\left\langle\sigma_{T}\right\rangle_{r}=0.65 \mathrm{~K}$ as compared to method $2\left\langle\sigma_{T}\right\rangle_{r}=1.3 \mathrm{~K}$, when using the same amount of computational time.

Figure 6 shows an example of the calculated spectral energy distribution for a method 2 model with few photons $\left(N_{\text {phot }}=10^{5}\right)$. The spectrum has a simple black-body characteristic, where the black-body parameters can be roughly fitted by a temperature of $800 \mathrm{~K}$ and an effective radius of $9.5 R_{\star}$. There is a slight excess in the blue part $(\lambda<1 \mu \mathrm{m})$ with respect to $9.5^{2} \pi B_{\lambda}(800 \mathrm{~K})$. Analysis shows that this part of the spectrum is dominated by stellar photons which are at least scattered once by the dust envelope. The figure also demonstrates the Monte Carlo errors of the flux determination. The best accuracy is achieved in the thermal infrared around the maximum of the spectrum (this nice correlation between physical importance and achieved accuracy is often found in Monte Carlo experiments). The noisiest results (errors of up to one order of magnitude for this few photon model) are obtained in the blue part of the spectrum. Here, there is actually a lot of photon energy present within the dust envelope, but this amount of energy is almost completely locked in. Only a tiny amount can escape. Additionally, the dust emission coefficient is very temperature-sensitive at these wavelengths, such that a small scatter in the temperature results in a large scatter of the spectral flux. Consequently, both Monte Carlo methods have some problems to arrive at accurate results in this spectral region.

For computations with $N_{\text {phot }}=1.6 \times 10^{7}$ and $2.4 \times 10^{6}$ for method 1 and 2 respectively, the agreement between the resulting fluxes of both models and the reference solution is excellent. However, the Monte Carlo method 1 is not capable to arrive at a reliable flux determination in the optical

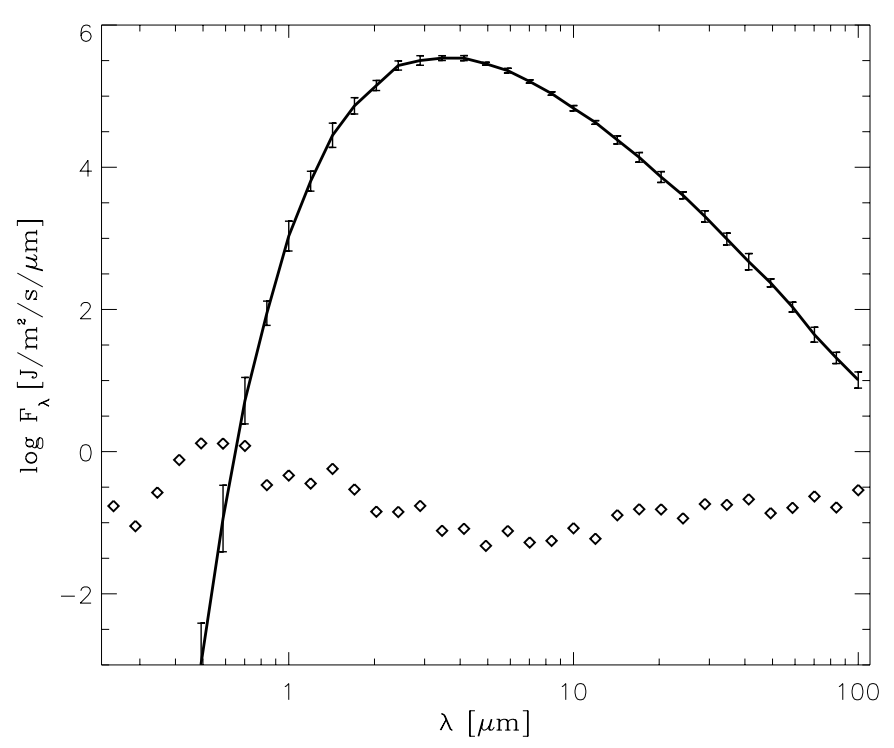

Fig. 6. Calculation of the Monte Carlo noise in the flux determination. We depict a method 2 model with few photons $\left(N_{\text {phot }}=10^{5}\right)$ in order to demonstrate the errors and related quantities. The full line with error-bars shows the mean values and standard deviations of the calculated flux, $\left\langle\log F_{\lambda}\right\rangle_{\theta}$ and $\sigma_{\log F_{\lambda}}$, respectively, considering 10 different escape directions. This standard deviation is estimated in a way similar to that of the temperature (see Eqs. (61) and (62)). The diamonds indicate the relative error $\sigma_{F_{\lambda}} /\left\langle F_{\lambda}\right\rangle_{\theta}$ on a logarithmic scale. The mean value of this quantity in wavelength space (here -0.74 , equivalent to $18 \%$ ) is chosen to represent the overall Monte Carlo noise of the flux determination as plotted in Fig. 8.

wavelength region where the photon packages have an extremely low probability to escape from the optically thick dust shell. Consequently, the numerical result for the escaping flux is often zero and the noise level is high. Method 2 is superior here. However method 1 is superior around the maximum and the far infrared, partly because the temperature structure is determined more precisely in the method 1 model. The mean relative Monte Carlo error over wavelength space $10^{\left\langle\log \left(\sigma_{F_{\lambda}} /\left\langle F_{\lambda}\right\rangle_{\theta}\right)\right\rangle_{\lambda}}(\lambda>0.4 \mu \mathrm{m})$ is $2.2 \%$ and $3.2 \%$ for method 1 and 2 , respectively.

Figures 7 and 8 show the dependence of the calculated Monte Carlo errors for the temperature and flux determination, respectively, as function of the number of photon packages used, in comparison to the expected $1 / \sqrt{N_{\text {phot }}}$ behaviour. A determination of the temperature structure better than $0.05 \%$ and a simultaneous determination of the spectral flux better than $1 \%$ can be achieved when using about $10^{8}$ or $2 \times 10^{7}$ photon packages with method 1 or 2 , respectively. Both methods require roughly the same computational time to achieve this accuracy (about $4000 \mathrm{~s}$ on a Cray T3E-1200 parallel computer using 16 processors).

\section{2D-applications}

\subsection{An optically thick dust cloud}

Figure 9 shows the resulting temperature structure in and around an opaque dust cloud close to a black body star. The cloud is assumed to be located at the pole between 


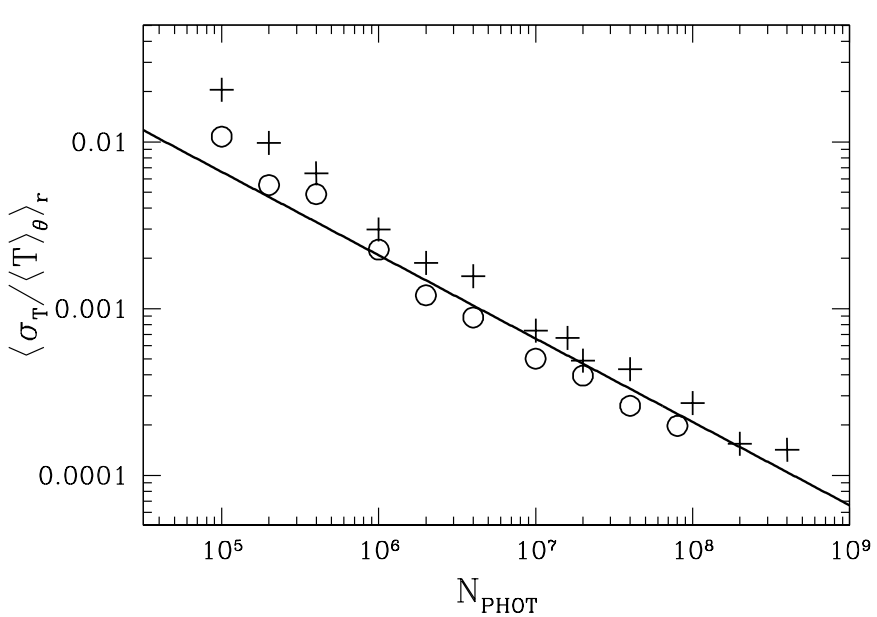

Fig. 7. Mean value of the relative Monte Carlo noise in the temperature determination $\sigma_{T} /\langle T\rangle_{\theta}$, considering the full radial extent of the model $r / R_{\star}=1 \ldots 300$, as function of the total number of used photon packages $N_{\text {phot }}$. Crosses and open circles mark method 1 and method 2 models, respectively. The full line shows the expected $1 / \sqrt{N_{\text {phot }}}$-dependence.

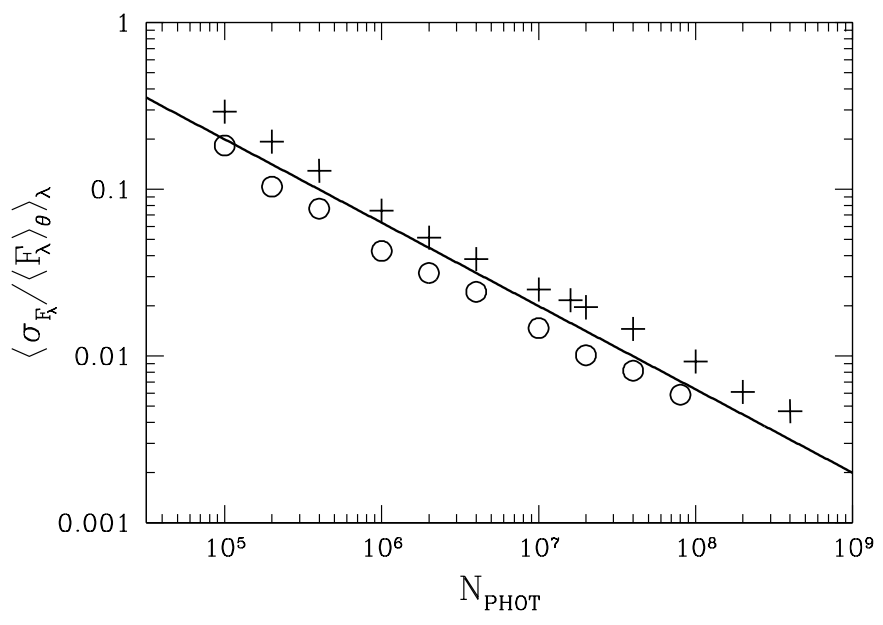

Fig. 8. Mean value of the relative flux noise $\sigma_{F_{\lambda}} /\left\langle F_{\lambda}\right\rangle_{\theta}$, considering wavelengths $\lambda=0.49 \mu \mathrm{m} \ldots 100 \mu \mathrm{m}$, as function of the total number of used photon packages $N_{\text {phot }}$. Crosses and open circles mark method 1 and method 2 models, respectively. The full line shows the expected $1 / \sqrt{N_{\text {phot }}}$-dependence.

$r=2.5 R_{\star}$ and $3.75 R_{\star}$ and between $\theta=0^{\circ}$ and $5.5^{\circ}$ with a radial optical depth of 10 at $1 \mu \mathrm{m}$. Outside of this cloud, the matter is assumed to be optically thin. The cloud is composed of amorphous carbon grains with a power-law size distribution between $0.001 \mu \mathrm{m}$ and $1 \mu \mathrm{m}$ of spectral index -3.5 . The optical data of amorphous carbon are taken from Rouleau $\&$ Martin (1991). For the underlying density structure, we assume a $1 / r^{2}$-law.

Due to the scattering and thermal re-emission, the dust cloud heats the matter ahead and aside of it - due to absorption, it shields and hence lowers the temperature of the matter in the cloud shadow. In particular, the calculation reveals the shape of the cloud's umbra and penumbra. The lowest temperatures (about $500 \mathrm{~K}$ ) occur close behind the cloud, whereas the highest temperatures (about $1100 \mathrm{~K}$ ) are present at the centre of the cloud's edge facing the star. The largest temperature gradients

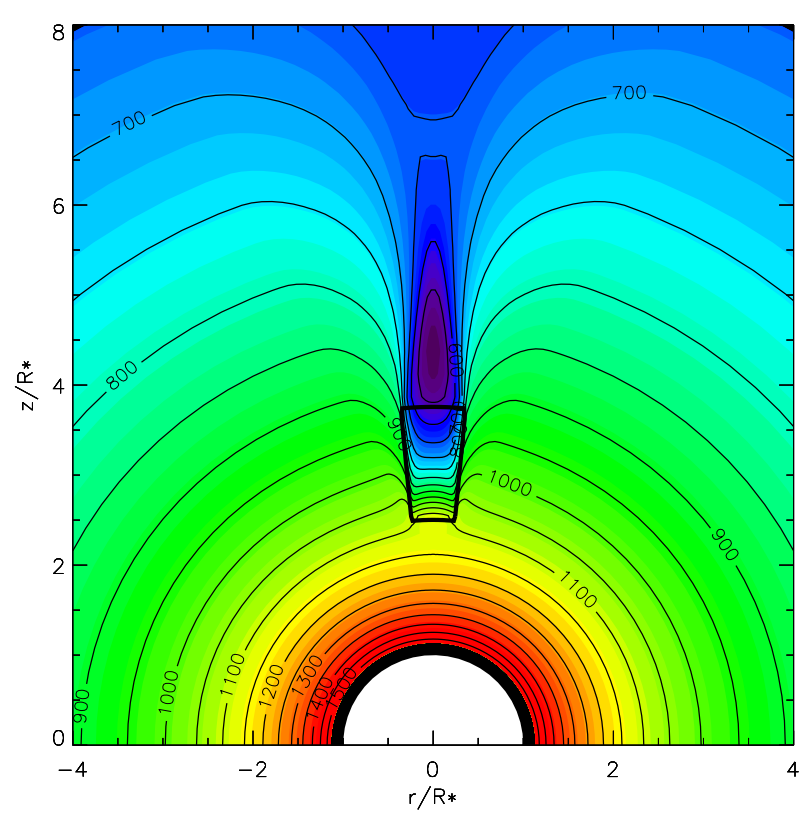

Fig. 9. Temperature map around an opaque dust cloud close to a black body star with $T_{\text {eff }}=2000 \mathrm{~K}\left(T_{\star}=2000.04 \mathrm{~K}\right)$. The colour map and contour lines show the calculated dust temperature structure. The dust cloud is surrounded by a thick solid line. The white circle represents the star. Black areas are not considered in the model.

are present within the cloud (both radially and tangentially), which is usually cooler inside than at its flanking edges.

Surprisingly, this cloud could hardly be detected by spectral analysis. The imposed changes of the spectral energy distribution in comparison to a model without the cloud are smaller than $0.5 \%$ at all wavelengths, except if the dust cloud is situated right in front of the star where it partly obscures the stellar disc. A black body fit to the residual cloud spectrum (total flux minus direct star light) at $\theta^{\text {esc }}=90^{\circ}$ (when the cloud is on top of the star) yields a fit temperature of about $1200 \mathrm{~K}$ which is in obvious contrast to the temperatures actually present in the cloud $(600 \mathrm{~K}$ to $1100 \mathrm{~K})$. A black body analysis is hence inappropriate for the determination of "cloud temperatures", at least in this case. First, the cloud is not at all isothermal, and second, the scattering leads to a blueing of the observed cloud spectrum.

The calculated spectral images of the cloud, for example at $\lambda=2.2 \mu \mathrm{m}$, show only a very faint feature originating from the illuminated warm surface of the cloud facing the star. The brightness of this feature, however, is so weak in comparison to the star (e.g. $B_{2.2 \mu \mathrm{m}}(1000 \mathrm{~K}) / B_{2.2} \mu \mathrm{m}(2000 \mathrm{~K}) \approx 3.7 \%$ that the image is simply dominated by the uniformly bright stellar disc.

\subsection{A hole in an optically thick dust shell}

Our last application considers an optically thick dust shell with a small conical opening around the pole (see Fig. 10). The dust shell extends radially from $2.5 R_{\star}$ and $4.5 R_{\star}$ and is restricted to latitude angles from $15^{\circ}$ to $180^{\circ}$. Again, the radial optical depth at $1 \mu \mathrm{m}$ is chosen to be 10 , and the cloud is assumed to be composed of amorphous carbon grains with a power-law size 


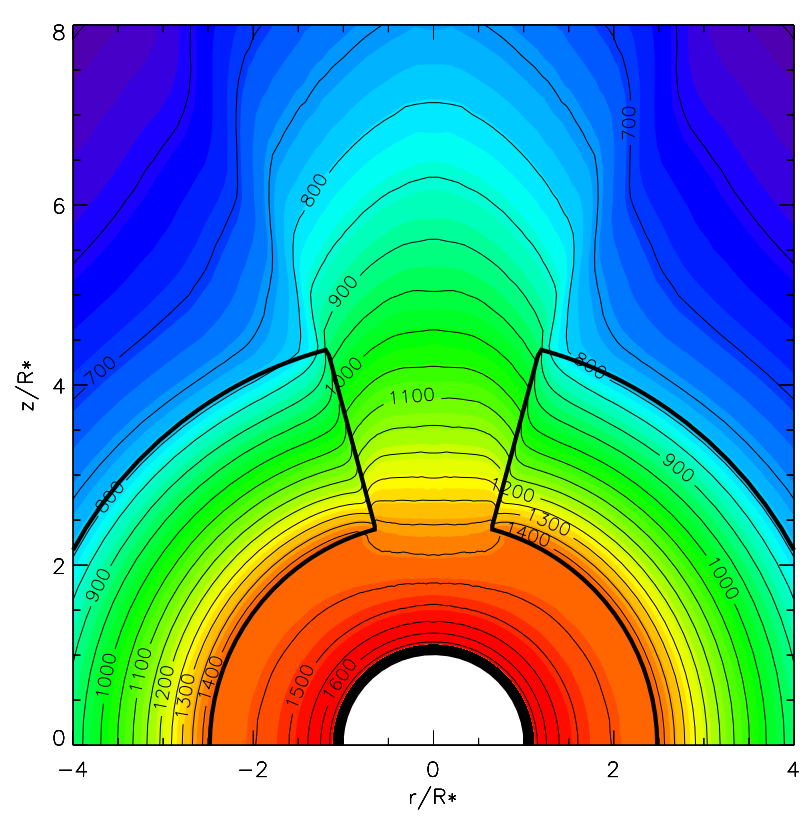

Fig. 10. Temperature map around a conical hole with a half opening angle of $15^{\circ}$ located at the pole of an opaque dust shell around a black body star with $T_{\text {eff }}=2000 \mathrm{~K}\left(T_{\star}=2067.9 \mathrm{~K}\right)$. The colour map with contour lines indicate the calculated dust temperature structure. The edges of the dust shell are marked by a thick solid black line. The white circle represents the star.

distribution between $0.001 \mu \mathrm{m}$ and $1 \mu \mathrm{m}$ of spectral index -3.5 . The underlying density structure is a $1 / r^{2}$-law. The effective temperature of the star is specified by $T_{\text {eff }}=2000 \mathrm{~K}$.

The calculation shows the strong increase of the temperatures at the location of the hole and outside of it in comparison with matter located at the same radial distance. The largest temperature gradients are present inside the dust shell whereas the temperature decreases only slowly with increasing radial distance along the hole.

A considerable fraction of the spectral flux escapes through the hole in the shell, thereby heating the flanking edges of the hole and the optically thin matter lying in-between. This directed flux results in an observable or even dominant feature in the calculated images, e.g. at $2.2 \mu \mathrm{m}$ (see Fig. 11). Analysis shows that the image mainly consists of thermal dust emission which is at least scattered once again by the dust envelope (58\%). Direct thermal dust emission (36\%) is the next important contribution whereas direct star light (1\%) and scattered star light (5\%) are less important. The feature in the image at the pole is hence a consequence of the higher dust temperatures inside the shell. Thermal emission from the hot interior is multiply scattered through and confined into the hole. Additionally, the hole may partly uncover the inner regions if the view direction is appropriate. The feature is even brighter at shorter wavelengths or larger optical depths.

These results are consistent with the finding of Men'shchikov et al. (2001) who constructed an empirical model for the dust envelope of IRC +10216 on the basis of high-resolution $J, H$, and $K$ speckle-interferometric images, suggesting that the brightest feature in the observations " $\mathrm{A}$ " is

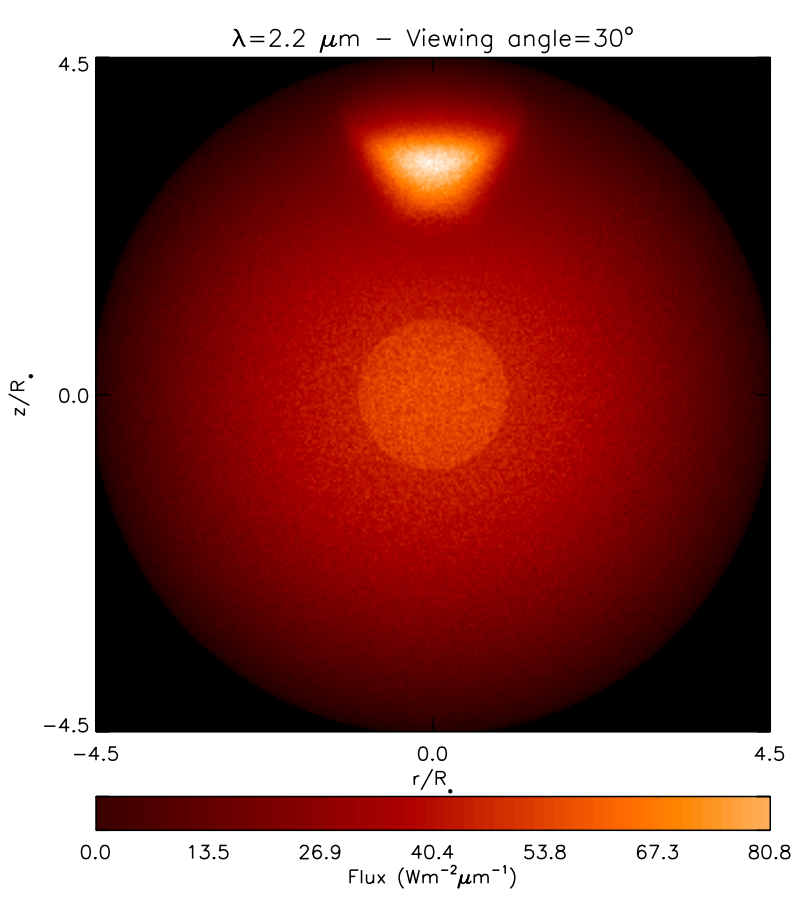

Fig. 11. Calculated spectral image of the model shown in Fig. 10 at $\lambda=2.2 \mu \mathrm{m}$. The flux values are given for an arbitrary distance of $1 R_{\star}$. The inclination angle between the equator plane and the observer direction is chosen to be $30^{\circ}\left(\theta^{\text {esc }}=60^{\circ}\right)$, i.e. the observer looks slightly into the hole of the dust shell which partly unfolds its hot interior. Consequently, the hole produces a bright feature around the pole.

rather to be identified with a hole in the dust shell ("outflow cavity") than with the star itself.

\section{Summary and future prospects}

A refined Monte Carlo method for the solution of multidimensional continuum radiative transfer problems in dusty media has been presented. The field of astrophysical application reaches from the (possibly cloudy) circumstellar environments of AGB and post AGB stars to the proto-planetary discs around young stellar objects (YSO). Other, e.g. interstellar or galactic applications are also conceivable.

We assume radiative equilibrium, LTE and angledependent coherent scattering. The opacities of the matter are assumed to be dominated by spherical dust grains which may have a spatially dependent size distribution. The dust opacities are explicitely temperature-independent, which considerably simplifies the problem of temperature determination. So far, the code can handle spherically symmetric (1D) and axisymmetric (2D) configurations. Full 3D problems have not been considered so far, but an extension to $3 \mathrm{D}$ is possible and straightforward.

In order to minimise the increase of the noise level in very optically thin or very optically thick situations, we have implemented several modifications of the standard Monte Carlo procedure:

1) The stellar and cell photon packages are systematically created and independently propagated through the dust 
envelope, thereby uniformly sampling the spatial origin as well as the frequency distribution of the emergent photons. This creation and propagation phase has to be carried out only once. The correct temperature structure of the medium in radiative equilibrium is found afterwards, applying an accurate iterative procedure. This temperature iteration is found to converge rapidly in optically thin as well as in optically thick situations and usually takes only a small fraction of the total computational time. However, it requires an origin-dependent storage of the primary Monte Carlo information, which needs a large amount of computer memory.

2) The condition of radiative equilibrium can be optionally formulated in terms of the mean intensity (method 1) according to (Lucy 1999) or by the equality of total emission and absorption in each cell (method 2). The determination of the temperature structure by mean intensities works particularly well for optically thin cells $(\tau=0)$, thereby solving the problems of standard Monte Carlo methods which usually occur in "empty cells".

3) Concerning the treatment of the absorption in method 2 , we have invented several deterministic elements all along the photon trajectories. This procedure requires more computational time per photon package, but is found to converge also for optically thick problems $(\tau \gg 1)$.

4) An exact tracing of the cell boundaries has been found to be necessary in order to accurately model discontinuous opacity structures.

We have tested the results of our two Monte Carlo methods against analytical solutions and reference solutions provided by a deterministic code for simple spherically symmetric cases. The Monte Carlo results agree well with the reference solutions concerning both temperature and flux determination. By increasing the number of photon packages, the Monte Carlo temperature noise level can easily be pushed below $1 \mathrm{~K}$, making the results practically indistinguishable from deterministic methods. The temperature noise is on a similar level in optically thin and optically thick regions. The noise level in the flux determination is a little larger (typically $1 \%$ ), especially in spectral regions far away from the maximum of the spectral energy distribution.

Some simple physical applications of our Monte Carlo radiative transfer code to the temperature structure and spectral appearance of dust clouds and small holes in dust shells have been presented. The temperature gradients (both radial and tangential) in isolated, optically thick dust clouds have been found to be remarkably large, especially within the surface layers, whereas the cloud cores are shielded and cool. Consequently, simple estimations of "cloud temperatures" on the basis of black body fits to observed spectra can be misleading. The spectral appearance of isolated dust clouds is generally found to be rather dark. The resulting cloud signatures in the calculated $K$-images are very faint in comparison to the star. In contrast to the clouds, an opening in an optically thick dust shell produces a bright feature in the images which can even dominate the spectral appearance of the star in certain view directions just the opposite of what could be expected. This finding is in general agreement with the conclusions of Men'shchikov et al. (2001) who suggested an "outflow cavity" to be responsible for the brightest feature (the "component A") in IR speckleinterferometric images of IRC+10216.

In summary, Monte Carlo methods in radiative transfer are easy to understand, flexible, and applicable to many physical problems in astronomy. Considering the ever increasing performance of current and future supercomputers, Monte Carlo methods become a more and more attractive tool not only for the purpose of the interpretation of observations, but also as a basic constituent of multi-dimensional, time-dependent modelling in astrophysics. We intend to apply the presented Monte Carlo method for the time-dependent modelling of multidimensional dust formation in the circumstellar environments of late-type stars, taking advantage of the method's capability to model accurately discontinuous opacity structures related to clumpy media. These models shall investigate the possibility of large-scale self-organisation and structure formation processes to occur as a consequence of radiative/thermal instabilities inherent in the dust formation process (Woitke et al. 2000). First models have shown positive results (Woitke 2001). We furthermore intend to analyse the proto-planetary discs of YSOs which requires a radiative transfer code which is capable of handling very large optical depths, at least along certain escape directions.

Acknowledgements. We warmly thank Thibaut Le Bertre for his engagement to provide us with a reference solution of the optically thick, spherically symmetric dust envelope as well as Jean-Marie Perrin for providing the Mie theory programs. We would like to thank the staff members of the Institut du Développement et des Ressources en Informatique Scientifique for their helpful assistance in the development of a parallelised version of method 2.

This work has been supported by the DFG, Sonderforschungsbereich 555, Komplexe Nichtlineare Prozesse, Teilprojekt B8, and by the DAAD in the PROCOPE program under grant $\mathrm{D} / 9822849$ and $\mathrm{F} / 99001$. The numerical computations were performed on the T3E parallel computer at the Konrad-Zuse-Zentrum für Informationstechnik Berlin, project bvpt17.

\section{Appendix A: Cell emission point}

We assume that the density dependence in the cell indexed by $\xi$ can be factorised according to $\rho(\boldsymbol{r})=\rho_{r}^{\xi}(r) \cdot \rho_{\theta}^{\xi}(\theta) \cdot \rho_{\varphi}^{\xi}(\varphi)$, where clearly in case of axisymmetry $\rho_{\varphi}^{\xi}(\varphi)=1$. Any further steps require a specification of the functions $\rho_{r}^{\xi}$ and $\rho_{\theta}^{\xi}$.

The most simple case occurs when we put $\rho_{\theta}^{\xi}=1$ and $\rho_{r}^{\xi}=$ $1 / r^{2}$, i.e. when we refrain from angular density dependencies and assume a $1 / r^{2}$-law inside each cell. In this case, the correct relations are found to be

$$
\begin{aligned}
& r=r_{n}+z_{1}\left(r_{n+1}-r_{n}\right) \\
& \cos \theta=\cos \theta_{k}+z_{2}\left(\cos \theta_{k+1}-\cos \theta_{k}\right) \\
& \varphi=2 \pi z_{3} .
\end{aligned}
$$

Other relations can be derived for other density functions. For example, Eq. (A.1) can be relaxed to the case 
$\rho_{r}^{\xi}=A_{\xi}+B_{\xi} / r^{2}$, where the radial density dependence is described by a general function with two individual fit coefficients, $A_{\xi}$ and $B_{\xi}$, for each cell. In this case $r$ can be obtained via the relation

$z_{1}=\frac{A_{\xi}\left(r^{3}-r_{n}^{3}\right)+3 B_{\xi}\left(r-r_{n}\right)}{A_{\xi}\left(r_{n+1}^{3}-r_{n}^{3}\right)+3 B_{\xi}\left(r_{n+1}-r_{n}\right)}$.

Equation (A.4) cannot be inverted analytically to give $r=r\left(z_{1}\right)$, but the solution can be found by a fast Newton-Raphson iteration. For consistency, the same fit functions $\rho_{r}^{\xi}$ and $\rho_{\theta}^{\xi}$ must be used for the calculation of the cell luminosity (Eq. (12)) and the calculation of the opacity for the optical depth integration.

\section{Appendix B: Scattering angle}

The fraction of intensity which is scattered from an initial direction $\widehat{\boldsymbol{n}}$ into the new direction $\widehat{\boldsymbol{n}}^{\prime}$ within solid angular interval $\mathrm{d} \widehat{\boldsymbol{n}}^{\prime}$ is proportional to $\kappa_{\lambda}^{\mathrm{sca}}(\boldsymbol{r}) \frac{1}{4 \pi} g_{\lambda}\left(\boldsymbol{r}, \widehat{\boldsymbol{n}}, \widehat{\boldsymbol{n}}^{\prime}\right) \mathrm{d} \widehat{\boldsymbol{n}}^{\prime}$. For spherical particles, the phase function depends only on the scattering angle $\vartheta \in[0, \pi)\left(\cos \vartheta=\widehat{\boldsymbol{n}} \cdot \widehat{\boldsymbol{n}}^{\prime}\right)$. Since $\kappa_{\lambda}^{\text {sca }}$ and $g_{\lambda}$ are assumed to be constant within one cell $\xi$, the probability of being scattered within $[\vartheta, \vartheta+\mathrm{d} \vartheta)$ in the cell indexed by $\xi$ is

$P^{\xi}(\vartheta) \mathrm{d} \vartheta=\frac{1}{2} g_{\lambda}^{\xi}(\vartheta) \sin \vartheta \mathrm{d} \vartheta$

The knowledge of $g_{\lambda} \xi(\vartheta)$ is usually restricted to a finite set of sampling points $\vartheta_{1}, \vartheta_{2}, \ldots, \vartheta_{I}$. The choice of $\vartheta$ can then be done via a (pseudo) random number $z_{1}$ uniformally distributed in $[0,1)$ according to

$\vartheta=\vartheta_{i-1}+\left(\vartheta_{i}-\vartheta_{i-1}\right) \frac{z_{1}-F_{i-1}}{F_{i}-F_{i-1}}$,

where

$$
F_{i}=\frac{\sum_{j=1}^{i} g_{\lambda}{ }^{\xi}\left(\vartheta_{j}\right) \sin \vartheta_{j}}{\sum_{j=1}^{I} g_{\lambda} \xi\left(\vartheta_{j}\right) \sin \vartheta_{j}}
$$

and $F_{i-1} \leq z_{1}<F_{i}$.
The choice of the new direction $\widehat{\boldsymbol{n}}^{\prime}$ is completed by specifying the latitude scattering angle $\varphi$ by means of another random number $z_{2}$ according to $\varphi=2 \pi z_{2}$.

\section{References}

Asplund, M., Ludwig, H.-G., Nordlund, Å., \& Stein, R. F. 2000, A\&A, 359,669

Bjorkman, J. E., \& Wood, K. 2001, ApJ, 554, 615

Cashwell, E. D., \& Everett, C. J. 1959, A Practical Manual on the Monte Carlo Method for Random Walk Problems (New York, Pergamon Press)

Fischer, O., Henning, Th., \& Yorke, H. W. 1994, A\&A, 284, 187

Gordon, K. D., Misselt, K. A., Witt, A. N., \& Clayton, G. C. 2001 , ApJ, 551, 269

Haniff, C. A., \& Buscher, D. F. 1998, A\&A, 334, L5

Hogerheidje, M. R., \& van der Tak, F. F. S. 2000, A\&A, 362, 697

Knuth, D. E. 1981, The Art of Computer Programming, Seminumerical Algorithms (2nd ed.), 2, 130

Levèvre, J., Bergeat, J., \& Daniel, J.-Y. 1982, A\&A, 114, 341

Leung, C. M. 1976, JQRST, 16, 559

Lucy, L. B. 1999, A\&A, 344, 282

Lopez, B., Mékarnia, D., \& Lefèvre, J. 1995, A\&A, 296, 752

Men'shchikov, A. B., Balega, Y., Blöcker, T., Osterbart, R., \& Weigelt, G., A\&A, 368, 497

Numerical Recipies: The Art of Scientific Computing, 1986 (Cambridge University Press)

Och, S. R., Lucy, L. B., \& Rosa, M. R. 1998, A\&A, 336, 301

O’Dell, C. R., \& Handron, K. D. 1996, AJ, 111, 1630

Rouleau, F., \& Martin, P. G. 1991, ApJ, 377, 526

Sedlmayr, E. 1993, in Molecules in the Stellar Environment, Proceedings of IAU Colloq. No. 146 held at Copenhagen, 1993, ed. U. G. Jorgensen, 440

Shara, M. M., Zurek, D. R., Williams, R. E., et al. 1997, AJ, 114, 258

Spaans, M. 1996, A\&A, 307, 271

Tuthill, P. G., Monnier, J. D., Danchi, W. C., \& Lopez, B. 2000, AJ, 543,284

Weigelt, G., Balega, Y., Blöcker, T., et al. 1998, A\&A, 333, L51

Witt, A. N. 1977, ApJS, 35, 1

Wiscombe, W. J. 1980, Appl. Opt., 19, 1505

Woitke, P., Sedlmayr, E., \& Lopez, B. 2000, A\&A, 358, 665

Woitke, P. 2001, Rev. Mod. Astron., 14, 185

Wolf, S., Henning, Th., \& Stecklum, B. 1999, A\&A, 349, 839

Yusef-Zadeh, F., \& Morris, M. 1984, ApJ, 278, 186 